\title{
44. ISOTOPE AND TRACE ELEMENT GEOCHEMISTRY OF EOCENE AND OLIGOCENE FORAMINIFERS FROM SITE 748, KERGUELEN PLATEAU1
}

\author{
James C. Zachos, ${ }^{2}$ William A. Berggren, ${ }^{3}$ Marie-Pierre Aubry, ${ }^{3,4}$ and Andreas Mackensen ${ }^{5}$
}

\begin{abstract}
Stable carbon and oxygen isotope analyses were conducted on well-preserved planktonic and benthic foraminifers from a continuous middle Eocene to Oligocene sequence at Ocean Drilling Program (ODP) Site 748 on the Kerguelen Plateau. Benthic foraminifer $\delta^{18} \mathrm{O}$ values show a $1.0 \%$ increase through the middle and upper Eocene, followed by a rapid $1.2 \%$ increase in the lowermost Oligocene $(35.5 \mathrm{Ma})$. Surface-dwelling planktonic foraminifer $\delta^{18} \mathrm{O}$ values increase in the lowermost Oligocene, but only by $0.6 \%$ whereas intermediate-depth planktonic foraminifers show an increase of about $1.0 \%$. Benthic foraminifer $\delta^{13} \mathrm{C}$ values increase by $0.9 \%$ in the lowermost Oligocene at precisely the same time as the large $\delta^{18} \mathrm{O}$ increase, whereas planktonic foraminifer $\delta^{13} \mathrm{C}$ values show little or no change.

Site 748 oxygen isotope and paleontological records suggest that southern Indian Ocean surface and intermediate waters underwent significant cooling from the early to late Eocene. The rapid $1.2 \%$ oxygen isotope increase recorded by benthic foraminifers just above the Eocene/Oligocene boundary represents the ubiquitous early Oligocene $\delta^{18} \mathrm{O}$ event. The shift here is unique, however, as it coincided with the sudden appearance of ice-rafted debris (IRD), providing the first direct link between Antarctic glacial activity and the earliest Oligocene $\delta^{18} \mathrm{O}$ increase. The $\delta^{18} \mathrm{O}$ increase caused by the ice-volume change in the early Oligocene is constrained by (1) related changes in the planktonic to benthic foraminifer $\delta^{18} \mathrm{O}$ gradient at Site 748 and (2) comparisons of late Eocene and early Oligocene planktonic foraminifer $\delta^{18} \mathrm{O}$ values from various latitudes. Both of these records indicate that $0.3 \%$ to $0.4 \%$ of the early Oligocene $\delta^{18} \mathrm{O}$ increase was ice-volume related.
\end{abstract}

\section{INTRODUCTION}

The transition from early Eocene global warmth to the cooler conditions of the Oligocene represents one of the more dramatic climatic changes in Earth's history. Paleontologic and oxygen isotope evidence indicate that the early Eocene was a period of exceptional warmth. For example, fossil remains of Eocene subtropical flora and vertebrates have been found in such high-latitude locations as Ellesmere Island in northern Baffin Bay (Estes and Hutchison, 1980; Wolfe, 1980). Similarly, fossils of temperate climate fauna and flora have been found in various locations along the Antarctic margin (Axelrod, 1984). Oxygen isotope records show early Eocene marine temperatures as high as $18^{\circ} \mathrm{C}$ on a seasonal basis at high latitudes (Stott et al., 1990) and deep-water temperatures as high as $15-17^{\circ} \mathrm{C}$ (e.g., Shackleton and Kennett, 1975; Miller et al., 1987a). During the middle Eocene to early Oligocene, following the early Eocene warmth, there was a decline in temperatures in the high latitudes and in the deep ocean, as reflected by changes in the faunal and floral records (e.g., Benson, 1975; Keller, 1983a; Aubry, 1983; Corliss, 1979; Boersma et al., 1987), and by a long term increase in the global benthic $\delta^{18} \mathrm{O}$ record (Douglas and Savin, 1975; Savin et al., 1975; Shackleton and Kennett, 1975; Savin, 1977; Miller et al., 1987a). This long-term $\delta^{18} \mathrm{O}$ trend was punctuated by several pronounced steps, with the most significant increases in the

\footnotetext{
${ }^{1}$ Wise, S. W., Jr., Schlich, R., et al., 1992. Proc. ODP, Sci. Results, 120: College Station, TX (Ocean Drilling Program).

2 Department of Geological Sciences, University of Michigan, Ann Arbor, MI 48109-1063, U.S.A.

${ }^{3}$ Woods Hole Oceanographic Institute, Woods Hole, MA 02543, U.S.A.

${ }^{4}$ URA 11, Université Claude Bernard, Department des Sciences de la Terre, 27-43 bd du 11 Novembre, 69622, Vilieurbanne Cedex, France.

5 Alfred-Wegener-Institut für Polar- und Meeresforschung, Postfach 120 161, Columbusstraße, D-2850 Bremerhaven, Federal Republic of Germany.
}

early-middle Eocene, late middle Eocene, late Eocene and just after the Eocene/Oligocene boundary. The last event was characterized by a rapid $>1.0 \%$ increase of $\delta^{18} \mathrm{O}$ as recorded by benthic foraminifers.

Much controversy has surrounded the significance of this early Oligocene $\delta^{18} \mathrm{O}$ event. Initially, some investigators suggested that the increase represented a $4^{\circ}-6^{\circ} \mathrm{C}$ cooling of deep-sea temperatures (e.g., Savin et al., 1975; Kennett and Shackleton, 1977; Kennett, 1977). This oxygen isotope shift was, however, also recorded by planktonic foraminifers from tropical regions where temperatures are thought to be constant, and therefore others argued that the shift primarily reflected an increase in global ice volume (Matthews and Poore, 1980; Poore and Matthews, 1984; Keigwin and Keller, 1984; Keigwin and Corliss, 1986; Prentice and Matthews, 1988). Moreover, if early Oligocene benthic foraminifers $\delta^{18} \mathrm{O}$ values were converted to paleotemperatures assuming an ice-free earth, bottom-water temperatures would have been several degrees colder than in the present-day ocean, a physical state that may be difficult to attain, especially on a warmer earth (Miller and Thomas, 1985; Miller et al., 1987a). The lack of more concrete evidence of a large early Oligocene ice sheet, however, such as widespread ice-rafted debris (IRD) (Kennett and Barker, 1990) or major coastal off-lap features in seismic records (Vail et al., 1977; Haq et al., 1987) has left this issue in doubt.

Controversy has also surrounded interpretation of Paleogene benthic foraminifer isotope records of deep-ocean circulation patterns. Some investigators have suggested that during periods characterized by low planetary temperature gradients, such as the Eocene, deep-water formation occurred primarily in low latitudes, possibly in semi-isolated evaporitic basins, rather than in polar regions where deep and intermediate waters formed during much of the Neogene (e.g., Chamberlin, 1906; Shackleton and Boersma, 1981; Brass et al., 1982; Prentice and Matthews, 1988; Kennett and Stott, 1990). In 


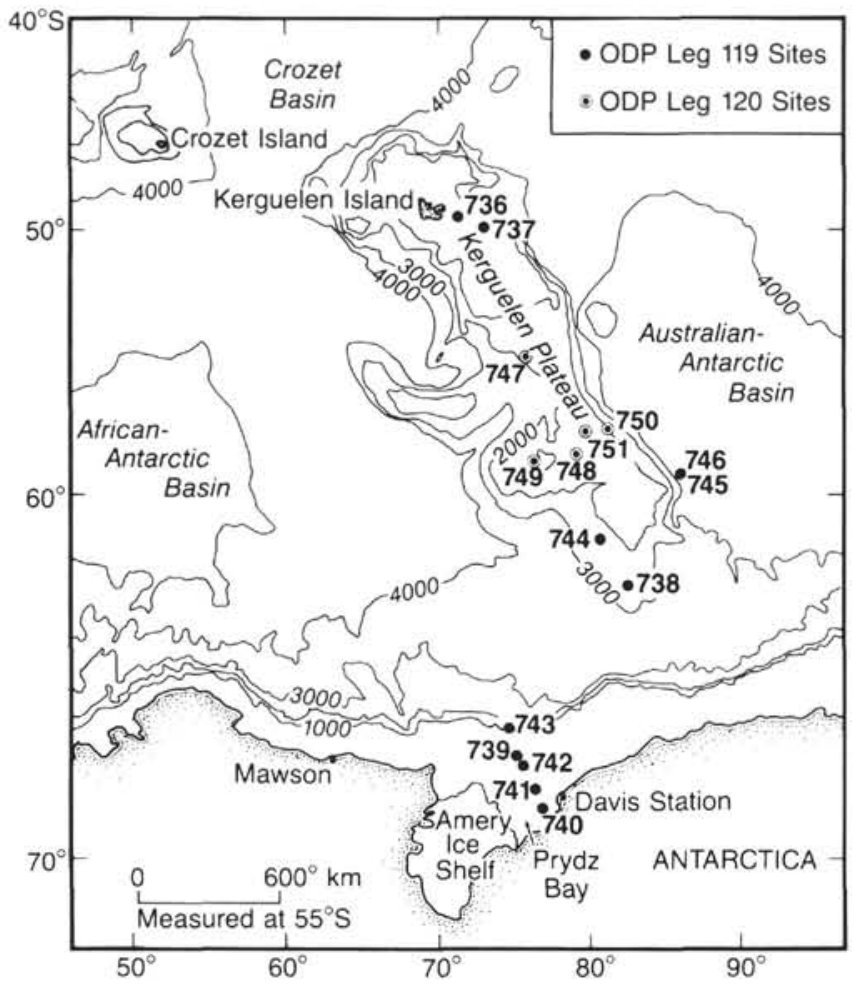

Figure 1. Map showing the Leg 119 and 120 sites on the Kerguelen Plateau and in Prydz Bay. Bathymetric contours in meters.

fact, some investigators considering meridional energy balances have suggested that such a circulation pattern might have been necessary to transfer the heat required to maintain the warm, early Eocene, high-latitude temperatures (e.g., Frakes and Kemp, 1973; Barron, 1987). The empirical record of Paleogene ocean circulation patterns and vertical water mass distributions, however, have been equivocal. For example, benthic foraminifer oxygen isotopic data from Maud Rise (Weddell Sea, southernmost Atlantic) suggest that warm saline bottom waters might have been present in the Southern Ocean during the Eocene and Oligocene (Kennett and Stott, 1990); yet comparisons of benthic foraminifer carbon isotope data from the Atlantic and central Pacific basins suggest that the primary source of bottom waters was near Antarctica during the Eocene, with the addition of a second deep-water source in the North Atlantic beginning in the early Oligocene (Miller et al., 1987b). The current ambiguity surrounding interpretation of Paleogene isotope records may result from the lack of spatial and vertical detail in isotope-based paleoclimate reconstructions. Few Paleogene sections have been recovered from climatically critical regions such as the high latitudes and equatorial regions.

The primary goal of this investigation was to provide a detailed geochemical record of late Eocene and Oligocene paleoenvironmental change in the high-latitude southern Indian Ocean. During Leg 120, a continuous lower middle Eocene to Miocene sequence of nannofossil ooze was recovered at Site 748 on Kerguelen Plateau. The paleodepth of the site through the Paleogene was upper bathyal $(-1000 \mathrm{~m})$ (Mackensen and Berggren, this volume). In this paper we present the results of a stable carbon, oxygen and strontium isotope and trace element investigation of planktonic and benthic foraminifers from Site 748 (Fig. 1). The oxygen isotope data provide information for reconstructing the evolution of surface and intermediate depth water temperatures at this location. The strontium isotope data are used for stratigraphic control over the Oligocene portion of the sequence, and the trace element data facilitate evaluation of foraminifer preservation. To assess the role of global ice-volume change on the Oligocene oxygen isotope record, Site 748 planktonic and benthic foraminifer $\delta^{18} \mathrm{O}$ values are compared with published data from other pelagic sites.

\section{ANALYTICAL TECHNIQUES}

To isolate foraminifers for geochemical analysis, about 15 $\mathrm{cm}^{3}$ of bulk sample was freeze-dried, weighed, disaggregated in Calgon solution, and wet-sieved through $63-\mu \mathrm{m}$ mesh sieve. After drying, individual planktonic and benthic foraminifers were handpicked from specific size-sorted samples. Specimens of the planktonic foraminifer Chiloguembelina were picked from the 88 - to $125-\mu \mathrm{m}$ size fraction, other species of planktonic foraminifers were picked from the $177-$ to $325-\mu \mathrm{m}$ fraction. Specimens of benthic foraminifer genera Cibicidoides spp. and Gyroidina spp. and ostracodes were taken from the $>150-\mu \mathrm{m}$ fraction. The microfossil samples were sonicated for $30 \mathrm{~s}$ in deionized water to remove adhering carbonate and clay particles. Suspended particles were decanted with a syringe. In final preparation for stable isotopic analysis, the foraminifers were roasted at $380^{\circ} \mathrm{C}$ under vacuum for 1 hour.

The stable isotopic analyses were conducted with a manually operated on-line gas extraction system coupled to a VG-602D mass-spectrometer, and an automated Kiel device carbonate preparation system coupled to a Finnegan MAT251 mass spectrometer. Under the manual system, $0.1-0.2 \mathrm{mg}$ samples were reacted in phosphoric acid at $55^{\circ} \mathrm{C}$; the resultant gas was subjected to a series of distillations to isolate the $\mathrm{CO}_{2}$ gas, which was then introduced into the VG-602D for analysis. Under the automated system, the reaction and distillation procedures were conducted with the automated Kiel Device, which reacted $0.03-0.08 \mathrm{mg}$ of sample with 4 drops of phosphoric acid at $75^{\circ} \mathrm{C}$ and isolated the $\mathrm{CO}_{2}$ in a single-step distillation. The $\mathrm{CO}_{2}$ was transferred to the MAT-251 mass spectrometer for measurement. The NBS-20 standard was measured on a daily basis to monitor instrument calibration and analytical accuracy. All isotope values are expressed in the $(\delta)$ notation where

$$
\delta(\%)=\left[\left(R_{\text {sample }} / R_{\text {standard }}\right)-1\right] \times 10^{3},
$$

relative to the PDB standard (Tables 1 and 2). Corrections were made for ${ }^{17} \mathrm{O}$ interference (Craig, 1957). The precision of replicate analyses was better than $\pm 0.1 \%$ for both oxygen and carbon.

Planktonic foraminifers from 15 upper Eocene and Oligocene samples were analyzed for their $\mathrm{Sr}$ isotopic composition to construct a strontium isotope record for stratigraphic purposes. Strontium isotope analyses were performed at the University of Michigan Radiogenic Isotope Laboratory. Mixed species of planktonic foraminifers were selected from the $>177-\mu \mathrm{m}$ sieved samples and sonicated in deionized water to remove adhering particles. About $0.5-1.0 \mathrm{mg}$ of each sample was dissolved in $1.0 \mathrm{ml}$ of $2.5 \mathrm{~N} \mathrm{HCl}$. The resulting solution was directly transferred to an ion exchange column by pipette. The ion exchange column contained $3.5 \mathrm{ml}$ of Biorad AG 1-X16 exchange resin. Following the exchange procedure, each sample was loaded onto a single-zone $\mathrm{Re}$ filament. The isotopic ratios were measured on a VG Sector multicollector mass spectrometer in peak "hopping" mode, using three Faraday collectors. Strontium isotope values are normalized to ${ }^{87} \mathrm{Sr} /{ }^{86} \mathrm{Sr}=0.1194$. Replicate analyses of the 
Table 1. Benthic foraminifer and ostracode stable carbon and oxygen isotope data, Site 748.

\begin{tabular}{|c|c|c|c|c|}
\hline $\begin{array}{l}\text { Core, section, } \\
\text { interval }(\mathrm{cm})\end{array}$ & $\begin{array}{l}\text { Depth } \\
\text { (mbsf) }\end{array}$ & Specimen & $\begin{array}{c}\delta^{18} \mathrm{O} \\
(\mathrm{PDB})\end{array}$ & $\begin{array}{c}\delta^{13} \mathrm{C} \\
(\mathrm{PDB})\end{array}$ \\
\hline \multicolumn{5}{|l|}{ 120-748B- } \\
\hline $9 \mathrm{H}-1,97$ & 67.57 & Cibicidoides & 1.94 & 0.82 \\
\hline $9 \mathrm{H}-1,97$ & 67.57 & Gyroidina & 2.39 & 0.75 \\
\hline $9 \mathrm{H}-1,97$ & 67.57 & Ostracoda & 2.44 & -2.59 \\
\hline $9 \mathrm{H}-2,100$ & 69.10 & Cibicidoides & 1.70 & 0.80 \\
\hline $9 \mathrm{H}-2,100$ & 69.10 & Gyroidina & 2.43 & 0.80 \\
\hline $9 \mathrm{H}-3,100$ & 70.60 & Cibicidoides & 1.72 & 0.90 \\
\hline $9 \mathrm{H}-3,100$ & 70.60 & Gyroidina & 2.13 & 0.61 \\
\hline $9 \mathrm{H}-4,100$ & 72.10 & Gyroidina & 2.47 & 0.50 \\
\hline $9 \mathrm{H}-4,100$ & 72.10 & Cibicidoides & 1.83 & 0.91 \\
\hline $9 \mathrm{H}-5,97$ & 73.57 & Cibicidoides & 1.88 & 0.73 \\
\hline $9 \mathrm{H}-5,97$ & 73.57 & Gyroidina & 2.25 & 0.57 \\
\hline $9 \mathrm{H}-6,98$ & 75.08 & Cibicidoides & 2.05 & 0.69 \\
\hline $9 \mathrm{H}-7,31$ & 75.91 & Cibicidoides & 2.16 & 0.80 \\
\hline $9 \mathrm{H}-7,31$ & 75.91 & Gyroidina & 2.62 & 0.71 \\
\hline $10 \mathrm{H}-1,100$ & 77.10 & Gyroidina & 2.48 & 0.46 \\
\hline $10 \mathrm{H}-1,100$ & 77.10 & N. umbonifera & 1.99 & 0.83 \\
\hline $10 \mathrm{H}-1,100$ & 77.10 & Ostracoda & 3.47 & 0.05 \\
\hline $10 \mathrm{H}-1,100$ & 77.10 & Cibicidoides & 1.97 & 0.93 \\
\hline $10 \mathrm{H}-2,100$ & 78.60 & Cibicidoides & 2.01 & 1.09 \\
\hline $10 \mathrm{H}-2,100$ & 78.60 & Gyroidina & 2.44 & 0.90 \\
\hline $10 \mathrm{H}-3,100$ & 80.10 & Cibicidoides & 2.08 & 1.13 \\
\hline $10 \mathrm{H}-3,100$ & 80.10 & Gyroidina & 2.66 & 0.86 \\
\hline $10 \mathrm{H}-4,100$ & 81.60 & Cibicidoides & 1.92 & 0.34 \\
\hline $10 \mathrm{H}-5,100$ & 83.10 & Cibicidoides & 1.93 & 0.70 \\
\hline $10 \mathrm{H}-5,100$ & 83.10 & Gyroidina & 2.35 & 0.33 \\
\hline $10 \mathrm{H}-6,30$ & 83.90 & Cibicidoides & 2.12 & 0.68 \\
\hline $11 \mathrm{H}-1,100$ & 86.60 & Cibicidoides & 2.01 & 0.60 \\
\hline $11 \mathrm{H}-2,100$ & 88.10 & Cibicidoides & 1.81 & 0.64 \\
\hline $11 \mathrm{H}-3,100$ & 89.60 & Cibicidoides & 2.39 & 0.61 \\
\hline $11 \mathrm{H}-3,100$ & 89.60 & Ostracoda & 4.07 & -0.44 \\
\hline $11 \mathrm{H}-4,100$ & 91.10 & Cibicidoides & 2.06 & 0.55 \\
\hline $11 \mathrm{H}-5,18$ & 91.78 & Cibicidoides & 2.08 & 0.46 \\
\hline $11 \mathrm{H}-5,100$ & 92.60 & Cibicidoides & 1.93 & 0.60 \\
\hline $11 \mathrm{H}-7,18$ & 94.78 & Cibicidoides & 2.21 & 0.55 \\
\hline $12 \mathrm{H}-1,100$ & 96.10 & Cibicidoides & 2.01 & 0.42 \\
\hline $12 \mathrm{H}-1,100$ & 96.10 & Gyroidina & 2.60 & 0.04 \\
\hline $12 \mathrm{H}-1,100$ & 96.10 & N. umbonifera & 2.00 & 0.24 \\
\hline $12 \mathrm{H}-1,100$ & 96.10 & Ostracoda & 3.45 & -0.45 \\
\hline $12 \mathrm{H}-3,18$ & 98.28 & Cibicidoides & 1.88 & 0.35 \\
\hline $12 \mathrm{H}-3,100$ & 99.10 & Cibicidoides & 1.76 & 0.10 \\
\hline $12 \mathrm{H}-3,100$ & 99.10 & Gyroidina & 2.27 & -0.04 \\
\hline $12 \mathrm{H}-4,100$ & 100.60 & Cibicidoides & 1.82 & 0.56 \\
\hline $12 \mathrm{H}-5,18$ & 101.28 & Cibicidoides & 2.20 & 0.39 \\
\hline $12 \mathrm{H}-5,18$ & 101.28 & Ostracoda & 3.35 & -0.21 \\
\hline $12 \mathrm{H}-5,100$ & 102.10 & Cibicidoides & 2.09 & 0.37 \\
\hline $12 \mathrm{H}-7,18$ & 104.28 & Cibicidoides & 1.94 & 0.79 \\
\hline $13 \mathrm{H}-1,100$ & 105.60 & Cibicidoides & 1.92 & 0.70 \\
\hline $13 \mathrm{H}-2,18$ & 106.28 & Cibicidoides & 1.87 & 0.96 \\
\hline $13 \mathrm{H}-2,100$ & 107.10 & Cibicidoides & 1.73 & 0.74 \\
\hline $13 \mathrm{H}-2,100$ & 107.10 & Ostracoda & 3.78 & 0.09 \\
\hline $13 \mathrm{H}-4,18$ & 109.28 & Cibicidoides & 2.01 & 0.81 \\
\hline $13 \mathrm{H}-4,18$ & 109.28 & Ostracoda & 3.78 & -0.04 \\
\hline $13 \mathrm{H}-4,100$ & 110.10 & Cibicidoides & 1.88 & 1.01 \\
\hline $13 \mathrm{H}-4,100$ & 110.10 & Gyroidina & 2.74 & 0.51 \\
\hline $13 \mathrm{H}-5,100$ & 111.60 & Cibicidoides & 2.24 & 1.28 \\
\hline $13 \mathrm{H}-6,181$ & 112.28 & Cibicidoides & 2.35 & 1.04 \\
\hline $14 \mathrm{H}-1,101$ & 114.20 & Gyroidina & 3.01 & -0.13 \\
\hline $14 \mathrm{H}-1,101$ & 114.20 & Ostracoda & 3.67 & -0.10 \\
\hline $14 \mathrm{H}-1,110$ & 115.20 & Cibicidoides & 2.51 & 1.68 \\
\hline $14 \mathrm{H}-1,110$ & 115.20 & Gyroidina & 3.03 & 0.80 \\
\hline $14 \mathrm{H}-1,110$ & 115.20 & Ostracoda & 3.54 & 0.45 \\
\hline $14 \mathrm{H}-1,138$ & 115.48 & Cibicidoides & 2.32 & 1.27 \\
\hline $14 \mathrm{H}-1,139$ & 115.49 & Cibicidoides & 2.41 & 1.40 \\
\hline $14 \mathrm{H}-1,139$ & 115.49 & Gyroidina & 2.95 & 0.62 \\
\hline
\end{tabular}

NBS-987 standard yielded a mean ratio of $0.710245 \pm 14(2 \sigma$ mean, $N=55$ ). Strontium isotope ratios are reported to five decimal places, as is the analytical precision. Carpenter et al. (in press) presents a more detailed explanation of the cation exchange procedure. For intercalibration purposes, the ${ }^{87} \mathrm{Sr} /$ ${ }^{86} \mathrm{Sr}$ ratios are also reported in the delta $(\delta)$ notation using the following equation:
Table 1. (continued)

\begin{tabular}{lllll}
\hline $\begin{array}{l}\text { Core, section, } \\
\text { interval }(\mathrm{cm})\end{array}$ & $\begin{array}{l}\text { Depth } \\
\text { (mbsf) }\end{array}$ & Specimen & $\begin{array}{c}\delta^{18} \mathrm{O} \\
(\mathrm{PDB})\end{array}$ & $\delta^{13} \mathrm{C}$ \\
$(\mathrm{PDB})$
\end{tabular}

120-748B- (Cont.)

\begin{tabular}{|c|c|c|c|c|}
\hline $14 \mathrm{H}-1,139$ & 115.49 & Ostracoda & 3.78 & 0.55 \\
\hline $14 \mathrm{H}-1,149$ & 115.59 & Gyroidina & 2.93 & 0.69 \\
\hline $14 \mathrm{H}-2,26$ & 115.85 & Cibicidoides & 1.20 & 0.71 \\
\hline $14 \mathrm{H}-2,26$ & 115.85 & Ostracoda & 2.74 & -0.01 \\
\hline $14 \mathrm{H}-2,39$ & 115.98 & Cibicidoides & 1.33 & 0.77 \\
\hline $14 \mathrm{H}-2,39$ & 115.98 & Ostracoda & 2.65 & -0.13 \\
\hline $14 \mathrm{H}-2,56$ & 116.15 & Cibicidoides & 1.14 & 0.9 \\
\hline $14 \mathrm{H}-2,56$ & 116.15 & Ostracoda & 2.80 & 0.29 \\
\hline $14 \mathrm{H}-2,95$ & 116.55 & Cibicidoides & 1.55 & 0.97 \\
\hline $14 \mathrm{H}-2,95$ & 116.55 & Ostracoda & 2.81 & 0.49 \\
\hline $14 \mathrm{H}-2,138$ & 116.98 & Cibicidoides & 1.35 & 0.86 \\
\hline $14 \mathrm{H}-3,138$ & 118.48 & Cibicidoides & 1.07 & 0.91 \\
\hline $14 \mathrm{H}-3,138$ & 118.48 & Ostracoda & 2.55 & 0.10 \\
\hline $14 \mathrm{H}-4,138$ & 119.98 & Cibicidoides & 1.28 & 0.76 \\
\hline $14 \mathrm{H}-5,138$ & 121.48 & Cibicidoides & 1.15 & 1.17 \\
\hline $14 \mathrm{H}-5,138$ & 121.48 & Ostracoda & 2.70 & 0.14 \\
\hline $14 \mathrm{H}-5,138$ & 121.48 & Cibicidoides & 1.25 & 1.17 \\
\hline $15 \mathrm{H}-1,20$ & 123.80 & Cibicidoides & 1.02 & 1.1 \\
\hline $15 \mathrm{H}-1,20$ & 123.80 & Gyroidina & 1.52 & 0.9 \\
\hline $15 \mathrm{H}-1,20$ & 123.80 & Ostracoda & 2.53 & 0.60 \\
\hline $15 \mathrm{H}-2,20$ & 125.30 & Cibicidoides & 0.89 & 1.21 \\
\hline $15 \mathrm{H}-2,20$ & 125.30 & Gyroidina & 1.42 & 0.65 \\
\hline $15 \mathrm{H}-3,44$ & 127.00 & Cibicidoides & 1.02 & 1.01 \\
\hline $15 \mathrm{H}-5,20$ & 129.80 & Cibicidoides & 0.92 & 1.38 \\
\hline $15 \mathrm{H}-5,20$ & 129.80 & Cibicidoides & 0.93 & 1.38 \\
\hline $15 \mathrm{H}-7,20$ & 132.80 & Cibicidoides & 1.23 & 1.29 \\
\hline $16 \mathrm{H}-2,18$ & 134.61 & Cibicidoides & 1.17 & 1.34 \\
\hline $16 \mathrm{H}-2,18$ & 134.61 & Gyroidina & 1.76 & 1.16 \\
\hline $16 \mathrm{H}-4,18$ & 137.61 & Cibicidoides & 1.48 & 1.20 \\
\hline $16 \mathrm{H}-4,18$ & 137.61 & G. beccariiformis & 1.42 & 1.25 \\
\hline $16 \mathrm{H}-4,18$ & 137.61 & Ostracoda & 2.81 & 0.32 \\
\hline $16 \mathrm{H}-4,18$ & 137.61 & Cibicidoides & 1.27 & 1.20 \\
\hline $16 \mathrm{H}-7,18$ & 141.33 & Cibicidoides & 1.09 & 1.21 \\
\hline $16 \mathrm{H}-7,18$ & 141.33 & G.beccariiformis & 1.08 & 0.90 \\
\hline $16 \mathrm{H}-7,18$ & 141.33 & Ostracoda & 2.68 & -0.53 \\
\hline $16 \mathrm{H}-7,18$ & 141.33 & Cibicidoides & 1.07 & 1.21 \\
\hline $17 \mathrm{H}-1,18$ & 142.78 & Ostracoda & 2.49 & -0.22 \\
\hline $17 \mathrm{H}-4,84$ & 147.90 & Cibicidoides & 0.71 & 1.04 \\
\hline $17 \mathrm{H}-5,18$ & 148.78 & Cibicidoides & 0.87 & 1.12 \\
\hline $17 \mathrm{H}-6,18$ & 150.28 & Cibicidoides & 1.00 & 0.91 \\
\hline $18 \mathrm{H}-1,100$ & 153.10 & Gyroidina & 1.31 & 0.61 \\
\hline $18 \mathrm{H}-2,44$ & 154.00 & Cibicidoides & 0.57 & 0.86 \\
\hline $18 \mathrm{H}-3,100$ & 156.10 & Cibicidoides & 0.69 & 0.78 \\
\hline $18 \mathrm{H}-3,100$ & 156.10 & Gyroidina & 1.16 & 0.60 \\
\hline $18 \mathrm{H}-5,100$ & 159.10 & Cibicidoides & 0.49 & 1.19 \\
\hline $18 \mathrm{H}-5,100$ & 159.10 & Ostracoda & 1.93 & 0.29 \\
\hline $19 \mathrm{H}-1,100$ & 162.60 & Cibicidoides & 0.23 & 1.29 \\
\hline $19 \mathrm{H}-1,100$ & 162.60 & Gyroidina & 1.05 & 1.20 \\
\hline $19 \mathrm{H}-1,100$ & 162.60 & Ostracoda & 1.74 & 0.54 \\
\hline $19 \mathrm{H}-3,100$ & 165.60 & Ostracoda & 1.46 & 0.56 \\
\hline $19 \mathrm{H}-5,100$ & 168.60 & Cibicidoides & 0.38 & 1.17 \\
\hline $20 \mathrm{H}-1,84$ & 171.90 & Cibicidoides & 0.58 & 0.4 \\
\hline
\end{tabular}

$120-748 \mathrm{C}$ -

\begin{tabular}{lllrr} 
10R-CC & 260.00 & Cibicidoides & -0.96 & 0.20 \\
14R-CC & 298.00 & Cibicidoides & -0.62 & -0.96 \\
21R-1, 72 & 350.80 & Cibicidoides & -0.49 & 0.24 \\
24R-1, 78 & 379.26 & G.beccariiformis & 0.10 & 1.63 \\
24R-CC & 380.00 & G.beccariiformis & 0.01 & 1.44 \\
25R-1, 57 & 388.53 & G.beccariiformis & 0.28 & 1.58 \\
25R-1, 81 & 388.83 & Gyroidina & -0.11 & 1.09 \\
25R-1, 124 & 389.22 & Gyroidina & -0.17 & 0.87 \\
\hline
\end{tabular}

$$
\delta^{87} \mathrm{Sr}=\left[\left({ }^{87} \mathrm{Sr} /{ }^{86} \mathrm{Sr}_{\text {sample }} /{ }^{87} \mathrm{Sr} /{ }^{86} \mathrm{Sr}_{\text {seawater }}\right)-1\right] \times 10^{5},
$$

where ${ }^{87} \mathrm{Sr} /{ }^{86} \mathrm{Sr}_{\text {seawater }}$ is the strontium isotopic composition of modern seawater (Table 3 ). The value obtained for seawater at the University of Michigan is $0.709179 \pm 14$.

To help assess planktonic foraminifer preservation, trace element concentrations were measured on 22 samples from 
Table 2. Planktonic foraminifer stable carbon and oxygen isotope data, Site 748.

\begin{tabular}{|c|c|c|c|c|}
\hline $\begin{array}{l}\text { Core, section, } \\
\text { interval }(\mathrm{cm})\end{array}$ & $\begin{array}{l}\text { Depth } \\
\text { (mbsf) }\end{array}$ & Specimen & $\begin{array}{c}\delta^{18} \mathrm{O} \\
(\mathrm{PDB})\end{array}$ & $\begin{array}{l}\delta^{13} \mathrm{C} \\
\text { (PDB) }\end{array}$ \\
\hline \multicolumn{5}{|l|}{$120-748 \mathrm{~B}-$} \\
\hline $11 \mathrm{H}-2,44$ & 87.50 & Mixed planktonic foraminifers & 1.40 & 0.87 \\
\hline $12 \mathrm{H}-1,129$ & 96.35 & Mixed planktonic foraminifers & 2.03 & 0.92 \\
\hline $12 \mathrm{H}-7,181$ & 04.28 & S. angiporoides & 2.10 & 1.08 \\
\hline $12 \mathrm{H}-7,441$ & 04.50 & C. cubensis & 1.35 & 1.48 \\
\hline $12 \mathrm{H}-7,441$ & 04.50 & S. angiporoides & 2.05 & 1.17 \\
\hline $13 \mathrm{H}-1,18$ & 104.78 & C. cubensis & 1.76 & 1.11 \\
\hline $13 \mathrm{H}-2,18$ & 106.28 & S. angiporoides & 1.72 & 1.28 \\
\hline $13 \mathrm{H}-2,76$ & 106.82 & C. cubensis & 1.33 & 1.45 \\
\hline $13 \mathrm{H}-3,18$ & 107.78 & C. cubensis & 1.17 & 1.65 \\
\hline $13 \mathrm{H}-4,18$ & 109.28 & C. cubensis & 0.99 & 1.52 \\
\hline $13 \mathrm{H}-4,84$ & 109.90 & Mixed planktonic foraminifers & 1.58 & 1.06 \\
\hline $13 \mathrm{H}-5,76$ & 111.32 & C. cubensis & 1.16 & 1.54 \\
\hline $13 \mathrm{H}-6,18$ & 112.28 & C. cubensis & 1.61 & 1.76 \\
\hline $13 \mathrm{H}-6,100$ & 113.10 & C. cubensis & 1.48 & 1.87 \\
\hline $14 \mathrm{H}-1,84$ & 114.90 & C. cubensis & 1.61 & 2.09 \\
\hline $14 \mathrm{H}-1,84$ & 114.90 & S. angiporoides & 2.09 & 1.58 \\
\hline $14 \mathrm{H}-1,138$ & 115.48 & C. cubensis & 1.83 & 2.05 \\
\hline $14 \mathrm{H}-1,138$ & 115.48 & S. angiporoides & 2.41 & 1.41 \\
\hline $14 \mathrm{H}-1,139$ & 115.49 & S. angiporoides & 2.22 & 1.62 \\
\hline $14 \mathrm{H}-1,149$ & 115.59 & S. angiporoides & 2.32 & 1.43 \\
\hline $14 \mathrm{H}-2,26$ & 115.85 & S. angiporoides & 1.36 & 1.50 \\
\hline $14 \mathrm{H}-2,39$ & 115.98 & S. angiporoides & 1.24 & 1.29 \\
\hline $14 \mathrm{H}-2,76$ & 116.32 & C. cubensis & 1.39 & 1.62 \\
\hline $14 \mathrm{H}-2,138$ & 116.98 & C. cubensis & 1.21 & 1.91 \\
\hline $14 \mathrm{H}-2,138$ & 116.98 & $S$. angiporoides & 1.29 & 1.25 \\
\hline $14 \mathrm{H}-3,44$ & 117.50 & C. cubensis & 1.01 & 1.94 \\
\hline $14 \mathrm{H}-3,138$ & 118.48 & C. cubensis & 0.96 & 1.85 \\
\hline $14 \mathrm{H}-3,138$ & 118.48 & S. angiporoides & 1.25 & 1.35 \\
\hline $14 \mathrm{H}-4,138$ & 119.98 & C. cubensis & 0.84 & 1.83 \\
\hline $14 \mathrm{H}-5,44$ & 120.50 & C. cubensis & 0.70 & 1.90 \\
\hline $14 \mathrm{H}-5,44$ & 120.50 & S. angiporoides & 1.25 & 1.22 \\
\hline $14 \mathrm{H}-5,76$ & 120.82 & C. cubensis & 1.00 & 1.86 \\
\hline $14 \mathrm{H}-5,138$ & 121.48 & C. cubensis & 0.87 & 1.96 \\
\hline $14 \mathrm{H}-5,138$ & 121.48 & S. angiporoides & 1.30 & 1.35 \\
\hline $14 \mathrm{H}-\mathrm{CC}$ & 123.60 & C. cubensis & 1.03 & 2.21 \\
\hline $15 \mathrm{H}-1,20$ & 123.80 & C. cubensis & 0.68 & 1.96 \\
\hline $15 \mathrm{H}-2,20$ & 125.30 & C. cubensis & 0.59 & 2.15 \\
\hline $15 \mathrm{H}-2,76$ & 125.82 & C. cubensis & 0.38 & 2.09 \\
\hline $15 \mathrm{H}-3,44$ & 127.00 & S. angiporoides & 0.87 & 1.47 \\
\hline $15 \mathrm{H}-5,76$ & 130.36 & C. cubensis & 0.43 & 2.08 \\
\hline $15 \mathrm{H}-7,44$ & 133.00 & C. cubensis & 0.64 & 1.87 \\
\hline $15 \mathrm{H}-7,44$ & 133.00 & Mixed planktonic foraminifers & 0.78 & 1.26 \\
\hline $16 \mathrm{H}-5,44$ & 139.33 & C. cubensis & 0.43 & 1.50 \\
\hline $16 \mathrm{H}-\mathrm{CC}$ & 142.60 & C. cubensis & 0.51 & 1.47 \\
\hline $17 \mathrm{H}-4,84$ & 147.90 & C. cubensis & 0.50 & 1.83 \\
\hline $17 \mathrm{H}-4,84$ & 147.90 & S. linaperta & 0.69 & 1.45 \\
\hline $17 \mathrm{H}-5,72$ & 149.28 & C. cubensis & 0.36 & 1.85 \\
\hline $18 \mathrm{H}-2,44$ & 154.00 & S. linaperta & 0.46 & 1.36 \\
\hline $18 \mathrm{H}-2,72$ & 154.28 & Chiloguembelina spp. & 0.34 & 1.54 \\
\hline $18 \mathrm{H}-5,72$ & 158.78 & Chiloguembelina spp. & 0.20 & 1.75 \\
\hline $19 \mathrm{H}-2,72$ & 163.78 & Chiloguembelina spp. & 0.06 & 1.70 \\
\hline $20 \mathrm{H}-1,84$ & 171.90 & Mixed planktonic foraminifers & 0.50 & 1.23 \\
\hline $20 \mathrm{H}-1,84$ & 171.90 & S. linaperta & 0.21 & 1.02 \\
\hline $20 \mathrm{H}-2,72$ & 173.28 & C. cubensis & 0.02 & 1.12 \\
\hline $24 \mathrm{X}-\mathrm{CC}$ & 206.40 & Chiloguembelina spp. & 0.70 & 0.48 \\
\hline \multicolumn{5}{|l|}{$120-748 \mathrm{C}$ - } \\
\hline $1 \mathrm{R}-2,76$ & 175.22 & C. cubensis & 0.25 & 1.44 \\
\hline $1 \mathrm{R}-\mathrm{CC}$ & 176.00 & C. cubensis & 0.63 & 1.30 \\
\hline $6 \mathrm{R}-\mathrm{CC}$ & 220.67 & Chiloguembelina spp. & 0.27 & 0.38 \\
\hline 10R-CC & 259.22 & Chiloguembelina spp. & -0.27 & 0.52 \\
\hline 10R-CC & 260.00 & Chiloguembelina spp. & -0.39 & 0.45 \\
\hline $14 \mathrm{R}-\mathrm{CC}$ & 298.00 & Chiloguembelina spp. & -0.38 & 0.04 \\
\hline 19R-CC & 332.50 & Mixed planktonic foraminifers & -0.37 & 2.26 \\
\hline 21R-CC & 351.50 & Chiloguembelina spp. & -0.22 & 1.95 \\
\hline 22R-CC & 361.00 & Chiloguembelina spp. & -0.39 & 1.29 \\
\hline 23R-CC, 18 & 369.16 & Chiloguembelina spp. & -0.17 & 1.60 \\
\hline $24 \mathrm{R}-1,78$ & 379.26 & Chiloguembelina spp. & -0.39 & 2.16 \\
\hline $24 \mathrm{R}-\mathrm{CC}$ & 380.00 & Chiloguembelina spp. & -0.52 & 2.74 \\
\hline $25 \mathrm{R}-1,57$ & 388.53 & Chiloguembelina spp. & -0.42 & 3.12 \\
\hline $25 \mathrm{R}-1,124$ & 389.22 & Chiloguembelina spp. & -0.80 & 2.67 \\
\hline
\end{tabular}

Table 3. Mixed planktonic foraminifer ${ }^{87} \mathrm{Sr} /{ }^{86} \mathrm{Sr}$ isotope data, Site 748.

\begin{tabular}{lrrrrr}
\hline $\begin{array}{c}\text { Core, section, } \\
\text { interval (cm) }\end{array}$ & $\begin{array}{r}\text { Depth } \\
\text { (mbsf) }\end{array}$ & ${ }^{87} \mathrm{Sr} /{ }^{86} \mathrm{Sr}$ & $\begin{array}{c} \pm 2 \sigma \\
\text { mean }\end{array}$ & ${ }^{87} \mathrm{Sr}$ & $\begin{array}{r} \pm 2 \sigma \\
\text { mean }\end{array}$ \\
\hline $120-748 \mathrm{~B}-$ & & & & & \\
& & & & & \\
$9 \mathrm{H}-1,40-44$ & 67.00 & 0.708188 & \pm 14 & -139.7 & \pm 1.9 \\
$11 \mathrm{H}-2,40-44$ & 87.50 & 0.708005 & \pm 14 & -165.5 & \pm 1.9 \\
$12 \mathrm{H}-7,40-44$ & 104.50 & 0.707946 & \pm 14 & -173.9 & \pm 1.9 \\
$13 \mathrm{H}-4,80-84$ & 109.90 & 0.707886 & \pm 16 & -182.3 & \pm 2.2 \\
$13 \mathrm{H}-6,18-22$ & 112.28 & 0.707824 & \pm 17 & -191.1 & \pm 2.3 \\
$14 \mathrm{H}-1,80-84$ & 114.90 & 0.707831 & \pm 14 & -190.1 & \pm 1.9 \\
$14 \mathrm{H}-2,134-138$ & 116.98 & 0.707796 & \pm 14 & -195.0 & \pm 1.9 \\
$14 \mathrm{H}-3,134-138$ & 118.48 & 0.707763 & \pm 14 & -199.7 & \pm 1.9 \\
$14 \mathrm{H}-4,134-138$ & 119.98 & 0.707808 & \pm 16 & -193.3 & \pm 2.2 \\
$14 \mathrm{H}-5,40-44$ & 120.50 & 0.707694 & \pm 17 & -209.4 & \pm 2.3 \\
$14 \mathrm{H}-5,134-138$ & 121.48 & 0.707696 & \pm 16 & -209.1 & \pm 2.2 \\
$15 \mathrm{H}-3,40-44$ & 127.00 & 0.707762 & \pm 14 & -199.8 & \pm 1.9 \\
$16 \mathrm{H}-5,40-44$ & 139.33 & 0.707730 & \pm 14 & -204.3 & \pm 1.9 \\
$18 \mathrm{H}-2,40-44$ & 154.00 & 0.707719 & \pm 14 & -205.9 & \pm 1.9 \\
$20 \mathrm{H}-3,80-84$ & 174.90 & 0.707769 & \pm 17 & -198.8 & \pm 2.3
\end{tabular}

Note: Error $(2 \sigma)$ is reported to the fifth decimal place.

Table 4. Mixed planktonic foraminifer $\mathrm{Mg} / \mathrm{Ca}, \mathrm{Sr} / \mathrm{Ca}, \mathrm{Fe} / \mathrm{Ca}$, and $\mathrm{Mn} / \mathrm{Ca}$ molar ratios, Site 748.

\begin{tabular}{lrrrrr}
\hline $\begin{array}{l}\text { Core, section, } \\
\text { interval (cm) }\end{array}$ & $\begin{array}{l}\text { Depth } \\
\text { (mbsf) }\end{array}$ & $\begin{array}{c}\mathrm{Mg} / \mathrm{Ca} \\
\left(\times 10^{3}\right)\end{array}$ & $\begin{array}{c}\mathrm{Sr} / \mathrm{Ca} \\
\left(\times 10^{3}\right)\end{array}$ & $\begin{array}{c}\mathrm{Fe} / \mathrm{Ca} \\
\left(\times 10^{3}\right)\end{array}$ & $\begin{array}{c}\mathrm{Mn} / \mathrm{Ca} \\
\left(\times 10^{3}\right)\end{array}$ \\
\hline $120-748 \mathrm{~B}-$ & & & & & \\
& & & & & \\
$9 \mathrm{H}-1,44$ & 67.00 & 3.62 & 1.30 & 0.69 & 0.18 \\
$10 \mathrm{H}-2,102$ & 83.10 & 2.91 & 1.29 & 1.34 & 0.17 \\
$11 \mathrm{H}-2,44$ & 87.50 & 3.40 & 1.29 & 1.30 & 0.24 \\
$12 \mathrm{H}-1,102$ & 96.10 & 2.96 & 1.31 & 1.07 & 0.20 \\
$12 \mathrm{H}-7,44$ & 104.50 & 2.57 & 1.27 & 0.86 & 0.12 \\
$13 \mathrm{H}-6,84$ & 112.28 & 2.78 & 1.31 & 0.83 & 0.09 \\
$14 \mathrm{H}-1,84$ & 114.90 & 3.04 & 1.33 & 0.73 & 0.09 \\
$14 \mathrm{H}-2,138$ & 116.98 & 2.99 & 1.31 & 0.68 & 0.12 \\
$14 \mathrm{H}-2,138 \mathrm{D}$ & 116.98 & 2.76 & 1.32 & 0.76 & 0.14 \\
$14 \mathrm{H}-2,138 \mathrm{D}$ & 116.98 & 2.60 & 1.35 & 0.45 & 0.14 \\
$14 \mathrm{H}-3,138$ & 118.48 & 3.25 & 1.46 & 1.14 & 0.12 \\
$14 \mathrm{H}-4,138$ & 119.98 & 3.12 & 1.40 & 0.74 & 0.11 \\
$14 \mathrm{H}-5,138$ & 121.48 & 2.72 & 1.49 & 0.58 & 0.10 \\
$14 \mathrm{H}-5,138 \mathrm{D}$ & 121.48 & 2.63 & 1.54 & 0.43 & 0.10 \\
$14 \mathrm{H}-5,138 \mathrm{D}$ & 121.48 & 2.79 & 1.47 & 0.65 & 0.08 \\
$15 \mathrm{H}-3,44$ & 127.00 & 2.59 & 1.53 & 0.60 & 0.05 \\
$15 \mathrm{H}-6,24$ & 131.30 & 2.83 & 1.50 & 0.78 & 0.09 \\
$16 \mathrm{H}-5,44$ & 139.33 & 2.70 & 1.46 & 0.38 & 0.05 \\
$17 \mathrm{H}-5,22$ & 148.78 & 2.53 & 1.34 & 0.27 & 0.04 \\
$18 \mathrm{H}-2,44$ & 154.00 & 2.75 & 1.37 & 0.42 & 0.07 \\
$18 \mathrm{H}-3,102$ & 156.10 & 3.09 & 1.44 & 1.13 & 0.06 \\
$19 \mathrm{H}-2,102$ & 164.10 & 2.66 & 1.42 & 0.27 & 0.07 \\
$20 \mathrm{H}-3,84$ & 174.90 & 3.33 & 1.39 & 0.55 & 0.07 \\
\hline
\end{tabular}

Site 748. Mixed species of planktonic foraminifers were analyzed for $\mathrm{Ca}, \mathrm{Sr}, \mathrm{Mg}, \mathrm{Mn}$, and $\mathrm{Fe}$ concentrations, using inductively coupled plasma emission spectrometry (ICP). Planktonic foraminifers were randomly picked from the $>150-\mu \mathrm{m}$ size fraction and cleaned (following the procedures described above). Typically, samples ranged from 1 to $3 \mathrm{mg}$ in weight. Samples were reacted in $1-\mathrm{ml}$ dilute $\mathrm{HCl} / \mathrm{HNO}_{3}$ and aspirated into a Leeman Labs Plasma-Spec III to be measured. Analytical precision was $\pm 1 \%$ for $\mathrm{Ca}, \pm 5.6 \%$ for $\mathrm{Mg}$, $\pm 5.1 \%$ for $\mathrm{Sr}, \pm 5.0 \%$ for $\mathrm{Fe}$, and $\pm 13.1 \%$ for $\mathrm{Mn}$, based on replicate analyses of a gravimetric standard. All trace element values are reported in mole ratios to $\mathrm{Ca}$ (Table 4).

To improve the resolution of the percent carbonate record generated on board ship during Leg 120, percent carbonate 
Table 5. Carbonate contents of whole-rock samples, Site 748.

\begin{tabular}{|c|c|c|c|c|}
\hline $\begin{array}{l}\text { Core, section, } \\
\text { interval }(\mathrm{cm})\end{array}$ & $\begin{array}{l}\text { Depth } \\
\text { (mbsf) }\end{array}$ & $\begin{array}{c}\%_{0} \mathrm{CaCO}_{3} \\
(\times 100)\end{array}$ & $\begin{array}{c}\% \mathrm{CaCO}_{3} \\
(\times 100)\end{array}$ & $\begin{array}{c}{ }_{0} \mathrm{CaCO}_{3} \\
(\times 100)\end{array}$ \\
\hline \multicolumn{5}{|l|}{$120-748 \mathrm{~B}-$} \\
\hline $8 \mathrm{H}-3,83-85$ & $* 60.93$ & 92 & & \\
\hline $8 \mathrm{H}-5,87-89$ & $* 63.97$ & 71 & & \\
\hline $8 \mathrm{H}-6,81-83$ & $* 65.41$ & 71 & & \\
\hline $9 \mathrm{H}-1,61-63$ & $* 67.21$ & 87 & & \\
\hline $9 \mathrm{H}-3,116-118$ & $\bullet 70.76$ & 86 & & \\
\hline $9 \mathrm{H}-4,127-129$ & $* 72.37$ & 81 & & \\
\hline $9 \mathrm{H}-6,22-24$ & $* 74.32$ & 91 & & \\
\hline $10 \mathrm{H}-1,125-127$ & $\bullet 77.35$ & 91 & & \\
\hline $10 \mathrm{H}-2,81-82$ & $* 78.41$ & 94 & & \\
\hline $10 \mathrm{H}-3,100-102$ & 80.10 & 96 & & \\
\hline $10 \mathrm{H}-4,100-102$ & 81.20 & 92 & & \\
\hline $10 \mathrm{H}-4,76-78$ & $* 81.36$ & 92 & & \\
\hline $10 \mathrm{H}-5,100-102$ & 83.10 & 91 & & \\
\hline $10 \mathrm{H}-6,41-43$ & $* 84.01$ & 90 & & \\
\hline $11 \mathrm{H}-1,100-102$ & 86.60 & 88 & & \\
\hline $11 \mathrm{H}-1,122-124$ & $* 86.82$ & 90 & & \\
\hline $11 \mathrm{H}-3,100-102$ & 89.60 & 82 & & \\
\hline $11 \mathrm{H}-3,126-128$ & $* 89.86$ & 84 & & \\
\hline $11 \mathrm{H}-4,100-102$ & 91.10 & 93 & & \\
\hline $11 \mathrm{H}-5,44-46$ & $* 92.04$ & 91 & & \\
\hline $11 \mathrm{H}-6,25-27$ & $* 93.35$ & 88 & & \\
\hline $11 \mathrm{H}-6,100-102$ & 94.10 & 87 & & \\
\hline $12 \mathrm{H}-1,100-102$ & 96.10 & 87 & & \\
\hline $12 \mathrm{H}-2,100-102$ & 97.60 & 91 & 87 & \\
\hline $12 \mathrm{H}-2,119-121$ & $* 97.79$ & 89 & & \\
\hline $12 \mathrm{H}-3,100-102$ & 99.10 & 93 & & \\
\hline $12 \mathrm{H}-3,129-131$ & $* 99.39$ & 90 & & \\
\hline $12 \mathrm{H}-4,100-102$ & 100.60 & 92 & 91 & \\
\hline $12 \mathrm{H}-4,136-138$ & $* 100.96$ & 92 & & \\
\hline $12 \mathrm{H}-5,100-102$ & 102.10 & 92 & & \\
\hline $12 \mathrm{H}-6,36-38$ & $* 102.96$ & 92 & 93 & 92 \\
\hline $13 \mathrm{H}-1,100-102$ & 105.60 & 83 & & \\
\hline $13 \mathrm{H}-2,100-102$ & 107.10 & & & \\
\hline $13 \mathrm{H}-4,57-59$ & $* 109.67$ & 76 & 78 & 74 \\
\hline $13 \mathrm{H}-5,100-102$ & 111.60 & 84 & & \\
\hline $13 \mathrm{H}-6,59-61$ & $* 112.69$ & 73 & & \\
\hline $14 \mathrm{H}-1,134-136$ & $* 115.44$ & 87 & 87 & 87 \\
\hline $14 \mathrm{H}-2,15-17$ & $* 115.75$ & 76 & 73 & 69 \\
\hline $14 \mathrm{H}-2,94-96$ & 116.54 & 91 & & \\
\hline $14 \mathrm{H}-4,68-70$ & $* 119.28$ & 94 & & \\
\hline $14 \mathrm{H}-5,101-103$ & 121.11 & 93 & & \\
\hline $15 \mathrm{H}-1,100-102$ & 124.60 & 95 & 93 & \\
\hline $15 \mathrm{H}-2,101-103$ & 126.11 & & & \\
\hline $15 \mathrm{H}-2,127-129$ & $* 126.37$ & 90 & 90 & 85 \\
\hline $15 \mathrm{H}-4,126-128$ & $* 129.36$ & 92 & 91 & 87 \\
\hline
\end{tabular}

Notes: Values marked by an asterisk in the first column were generated on board ship. All other values were generated in this study.

analyses were conducted on 50 additional bulk samples (Table 5). Each whole-rock sample was freeze dried and ground by mortar and pestle. Analyses were conducted on $0.3-0.5 \mathrm{~g}$ of sample using the carbonate "bombe" technique of Müller and Gastner (1971). The precision of replicate analyses was $\pm 3 \%$. Because the shipboard percent carbonate analyses were conducted using the coulometric technique (see Schlich, Wise, et al., 1989, "Explanatory Notes" chapter), replicate analyses were conducted on 12 samples analyzed on board ship with a resulting precision of $\pm 2.6 \%$.

\section{SITE LOCATION, LITHOLOGY, AND STRATIGRAPHY}

Site $748\left(58^{\circ} 26.45^{\prime} \mathrm{S}, 78^{\circ} 58.89^{\prime} \mathrm{E}\right)$ was drilled at a water depth of $1290 \mathrm{~m}$ in the western part of the Raggatt Basin on the Southern Kerguelen Plateau to a sub-bottom depth of $935 \mathrm{~m}$ (Fig. 1). An expanded sequence of Paleogene foraminifer nannofossil ooze was recovered from 60 to $400 \mathrm{mbsf}$. Core recovery above the middle Eocene was excellent $(\sim 100 \%)$, with no recognizable unconformities (see Berggren et al., this volume). Recovery rates below the middle Eocene were $<20 \%$ because of encounters with chert beds during drilling. Siliceous microfossils, a minor component of this sequence,
Table 5. (continued)

\begin{tabular}{|c|c|c|c|c|}
\hline $\begin{array}{l}\text { Core, section, } \\
\text { interval }(\mathrm{cm})\end{array}$ & $\begin{array}{l}\text { Depth } \\
\text { (mbsf) }\end{array}$ & $\begin{array}{c}\% \mathrm{CaCO}_{3} \\
(\times 100)\end{array}$ & $\begin{array}{c}\% \mathrm{CaCO}_{3} \\
(\times 100)\end{array}$ & $\begin{array}{c}{ }_{\%}^{\% \mathrm{CaCO}_{3}} \\
(\times 100)\end{array}$ \\
\hline \multicolumn{5}{|l|}{ 120-748B-(Cont.) } \\
\hline $15 \mathrm{H}-6,126-128$ & $* 132.36$ & 93 & 92 & 89 \\
\hline $15 \mathrm{H}-7,57-59$ & 133.17 & 94 & & \\
\hline $16 \mathrm{H}-1,100-102$ & 134.10 & 93 & & \\
\hline $16 \mathrm{H}-2,30-32$ & $* 134.73$ & 96 & & \\
\hline $16 \mathrm{H}-4,20-22$ & $\bullet 137.63$ & 96 & 96 & \\
\hline $16 \mathrm{H}-4,100-102$ & 138.43 & 96 & 95 & \\
\hline $16 \mathrm{H}-6,20-22$ & $* 140.63$ & 96 & 96 & 94 \\
\hline $16 \mathrm{H}-6,63-65$ & 141.06 & 94 & & \\
\hline $17 \mathrm{H}-2,18-22$ & 144.28 & 97 & 96 & \\
\hline $17 \mathrm{H}-2,130-132$ & $* 145.40$ & 96 & & \\
\hline $17 \mathrm{H}-4,130-132$ & $* 148.40$ & 96 & & \\
\hline $17 \mathrm{H}-5,100-102$ & 149.60 & 87 & & \\
\hline $17 \mathrm{H}-6,18-22$ & 150.28 & 94 & & \\
\hline $17 \mathrm{H}-6,130-132$ & ${ }^{*} 151.40$ & 95 & 96 & 90 \\
\hline $18 \mathrm{H}-1,18-22$ & 152.28 & 94 & 93 & \\
\hline $18 \mathrm{H}-1,100-102$ & 153.10 & 91 & 94 & \\
\hline $18 \mathrm{H}-2,100-102$ & 154.60 & 94 & 96 & \\
\hline $18 \mathrm{H}-2,130-132$ & $* 154.90$ & 96 & 97 & \\
\hline $18 \mathrm{H}-3,18-22$ & 155.28 & 97 & 93 & \\
\hline $18 \mathrm{H}-4,18-22$ & 156.78 & 92 & 95 & \\
\hline $18 \mathrm{H}-4,100-102$ & 157.60 & 95 & 97 & \\
\hline $18 \mathrm{H}-4,134-136$ & +157.94 & 95 & 95 & 94 \\
\hline $18 \mathrm{H}-5,18-22$ & 158.28 & 94 & 94 & \\
\hline $18 \mathrm{H}-5,100-102$ & 159.10 & 94 & 92 & \\
\hline $18 \mathrm{H}-6,18-22$ & 159.78 & 94 & 94 & \\
\hline $18 \mathrm{H}-6,100-102$ & 160.60 & 94 & & \\
\hline $18 \mathrm{H}-6,132-134$ & $* 160.92$ & 93 & & \\
\hline $19 \mathrm{H}-1,100-102$ & 162.60 & 91 & & \\
\hline $19 \mathrm{H}-2,100-102$ & 164.10 & 96 & & \\
\hline $19 \mathrm{H}-2,132-134$ & $\cdot 164.42$ & 92 & 92 & \\
\hline $19 \mathrm{H}-4,100-102$ & 167.10 & 93 & 92 & \\
\hline $19 \mathrm{H}-4,132-134$ & $* 167.42$ & 93 & & \\
\hline $19 \mathrm{H}-6,52-54$ & $* 169.62$ & 97 & & \\
\hline $20 \mathrm{H}-1,59-61$ & $\bullet 171.69$ & 95 & & \\
\hline $20 \mathrm{H}-2,100-102$ & 173.60 & 93 & & \\
\hline $20 \mathrm{H}-3,84-86$ & $\bullet 174.94$ & 96 & & \\
\hline $20 \mathrm{H}-4,100-102$ & 176.60 & 97 & & \\
\hline $20 \mathrm{H}-6,100-102$ & 179.60 & 96 & 95 & \\
\hline $20 \mathrm{H}-7,91-93$ & 181.01 & 98 & & \\
\hline \multicolumn{5}{|l|}{$120-748 \mathrm{C}$} \\
\hline $1 \mathrm{R}-1,77-79$ & $* 173.77$ & 96 & & \\
\hline IR-2, 99-101 & $* 175.49$ & 95 & & \\
\hline
\end{tabular}

first appear in abundance $(>5 \%)$ in the upper Eocene just below the Eocene/Oligocene boundary and persist throughout the Oligocene. The Eocene/Oligocene boundary was identified at 122 mbsf in Core 120-748B-14H. Traces of IRD first occur at 117 mbsf (i.e., above the Eocene/Oligocene boundary) and are found throughout the remaining Oligocene sequence. The debris consists mainly of angular quartz sand, altered feldspar, mica, and heavy minerals, with individual grains as large as $1 \mathrm{~mm}$ in diameter. The concentration of debris peaks between 115.45 and 115.95 mbsf. A more detailed description of the IRD interval lithology is given by Breza and Wise (this volume).

The Paleogene stratigraphy at Site 748 is based mainly on planktonic foraminifer and calcareous nannofossil zonations (Aubry, this volume; Berggren et al., this volume). The top of the Oligocene was placed at 63.4 to $65 \mathrm{mbsf}$, where the last appearance datum (LAD) of Globigerina euapertura was recorded. The lower/upper Oligocene boundary was placed between 83.9 and $85.6 \mathrm{mbsf}$, based on the LAD of Chiloguembelina cubensis; and the boundary between the Eocene and Oligocene was placed between 120.5 and 122.4 mbsf, based on the LAD of Globigerinatheka index. The LADs of the typical middle Eocene elements Acarinina collactea and A. primitiva 


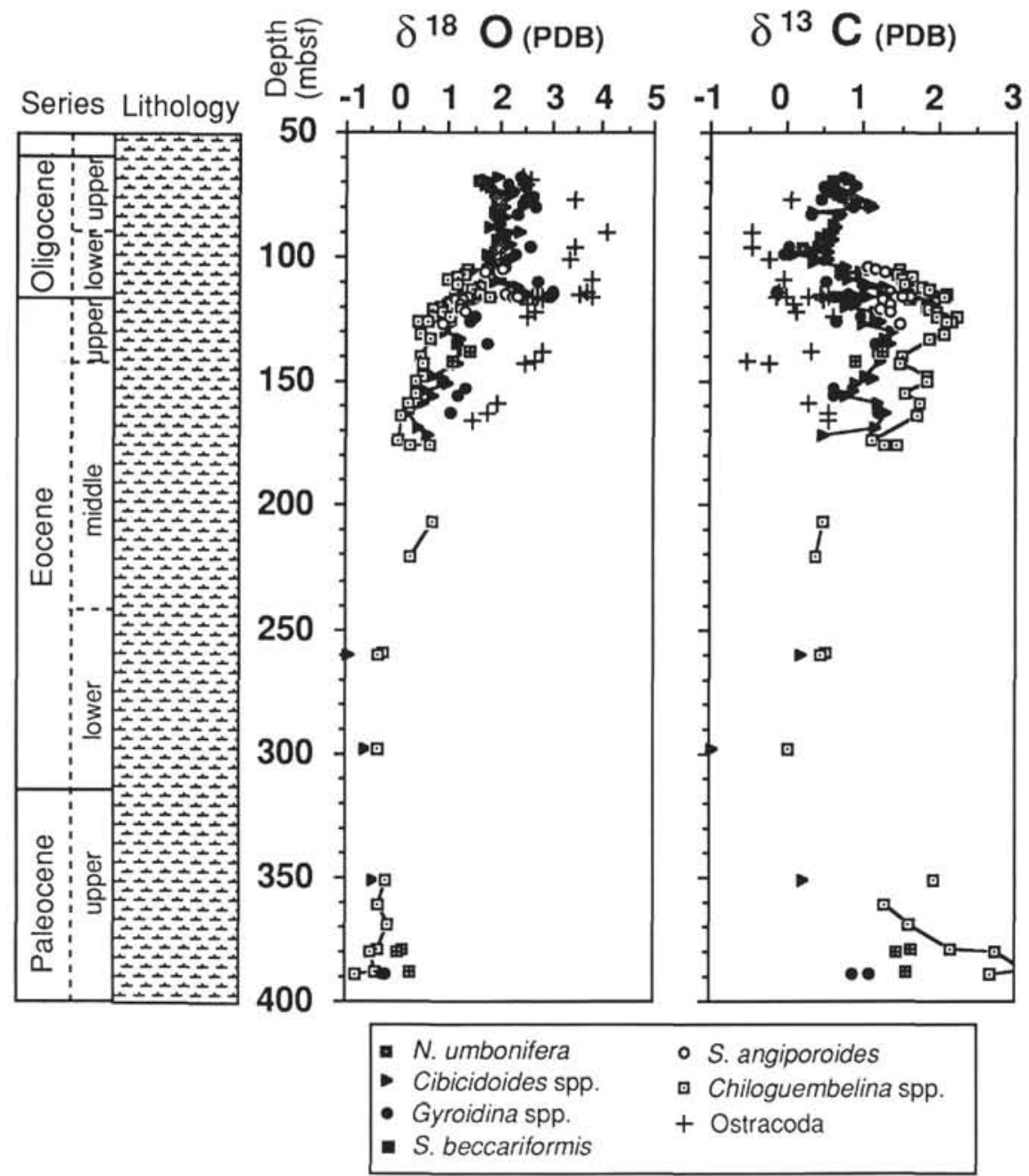

Figure 2. Stable oxygen and carbon isotope data of planktonic and benthic foraminifers and ostracodes plotted vs. depth, Site 748.

were recorded at 142 and 152.5 mbsf, respectively. The presence of all the characteristic upper Eocene and Oligocene planktonic foraminifer zones indicates that this sequence is continuous with no significant gaps or hiatuses at the present biostratigraphic resolution.

\section{RESULTS}

\section{Oxygen Isotopes}

The Cibicidoides $\delta^{18} \mathrm{O}$ values increase from $-0.5 \%$ to $1.5 \%$ over the lower to upper middle Eocene and then reverse at the middle/upper Eocene boundary, decreasing to $1.1 \%$ by the late Eocene (Figs. 2 and 3). In the lower Oligocene, from 118.5 to 115.5 mbsf, Cibidicoides show a $1.2 \%$ increase, with a peak value of $2.5 \%$ at 115.5 mbsf. Above this interval, still within the lower Oligocene, Cibicidoides $\delta^{18} \mathrm{O}$ values display a $0.5 \%$ decrease and then remain constant between 1.8 and $2.1 \%$ until 90 mbsf, where there is a slight increase to $2.5 \%$ o. From 90 to $70 \mathrm{mbsf}$, near the top of the Oligocene, the Cibicidoides $\delta^{18} \mathrm{O}$ values gradually decrease to $1.75 \%$. Gyroidina yielded $\delta^{18} \mathrm{O}$ compositions that were consistently offset by $0.57 \%$ ( \pm 0.11$)$ from Cibicidoides (Fig. 4). This offset between benthic foraminifer genera probably results from differences in disequilibrium vital effects with seawater (e.g., Shackleton et al., 1984). Ostracodes are consistently offset from Cibicidoides by $1.54 \%$
$( \pm 0.16)$, and therefore show a magnitude of change similar to the benthic foraminifers upsection (Figs. 3 and 4).

The $\delta^{18} \mathrm{O}$ values of planktonic foraminifer Chiloguembelina, mainly the species $C$. cubenis above the middle Eocene, are slightly lower than the values of Cibicidoides with two notable exceptions: (1) in the lower Eocene where the Chiloguembelina values are slightly higher than Cibicidoides values and (2) in the uppermost Eocene where the values are just $0.1 \%$ to $0.2 \%$ o lower than the Cibicidoides values (Fig. 2). Some species of Chiloguembelina are considered to have been near-surface dwellers because they usually yield the lowest $\delta^{18} \mathrm{O}$ values of late Eocene and early Oligocene planktonic foraminifers (Keller, 1983a; Poore and Matthews, 1984; Keigwin and Corliss, 1986). At Site 748 Subbotina angiporoides, an inferred intermediate depth dweller (e.g., Keller, 1983a; Poore and Matthews, 1984; Shackleton et al., 1985), yielded $\delta^{18} \mathrm{O}$ values that were generally higher than Chiloguembelina values and closer to Cibicidoides values, reflecting the lower temperatures of the intermediate-depth waters in which they reside. Chiloguembelina shows a small precursor enrichment at 126 mbsf in the upper Eocene, which essentially eliminates the $\delta^{18} \mathrm{O}$ gradient between Chiloguembelina and $S$. angiporoides and Cibicidoides. At 114 mbsf in the lower Oligocene both Chiloguembelina and $S$. angiporoides show pronounced $\delta^{18} \mathrm{O}$ increases of $0.6 \%$ and $1.0 \%$, respectively. As a result of the 


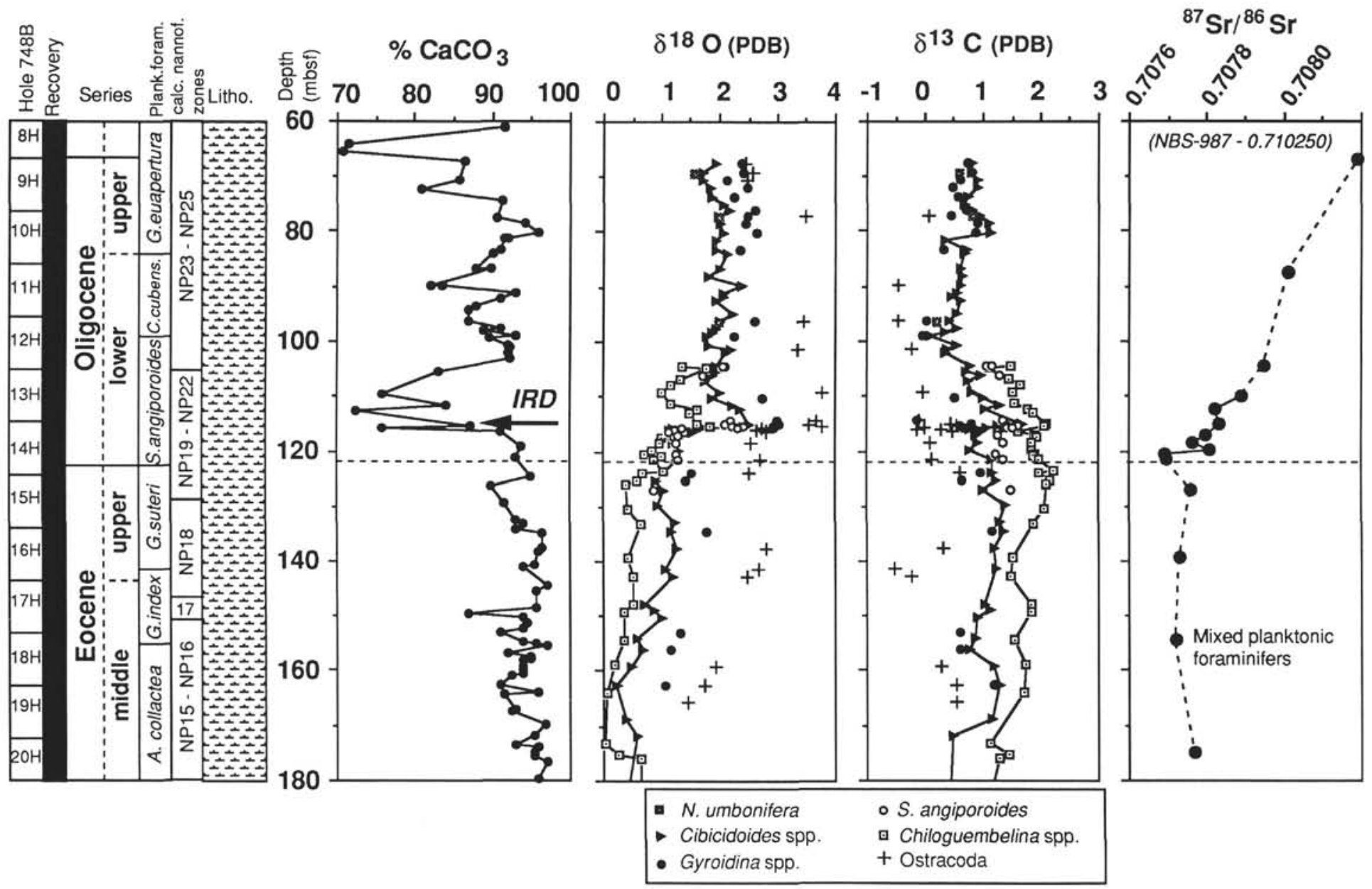

Figure 3. An expanded view of the Eocene/Oligocene boundary interval from the record presented in Figure 2. Bulk CaCO 3 contents and mixed planktonic foraminifer ${ }^{87} \mathrm{Sr} /{ }^{86} \mathrm{Sr}$ isotope data are included. Core recovery and lithology are shown to the left. 


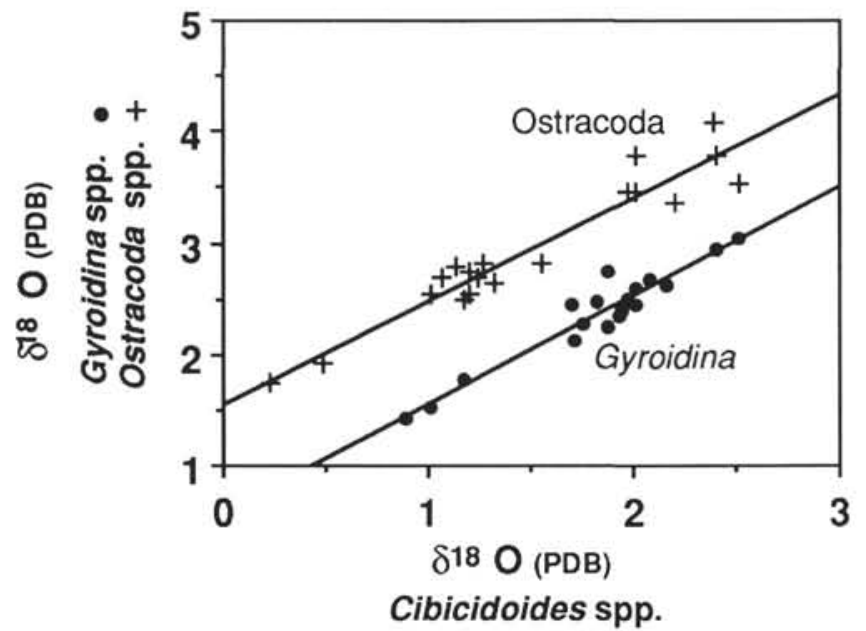

Figure 4. Gyroidina (indicated by a filled circle; $y=0.57+0.98 x$ ) and ostracode (indicated by a plus sign; $y=1.55+0.93 x$ ) $\delta^{18} \mathrm{O}$ values are plotted vs. Cibicidoides $\delta^{18} \mathrm{O}$ values for the interval from 60 to 180 mbsf. The correlation coefficient $R 2$ was equal to 0.924 for Gyroidina and 0.919 for the ostracodes.

smaller increase in Chiloguembelina the difference in $\delta^{18} \mathrm{O}$ values between Chiloguembelina and Cibicidoides in the early Oligocene is about $0.75 \%$, which is similar to that of the lower Eocene-upper Eocene at this location. The lower Oligocene $\delta^{18} \mathrm{O}$ increase coincides with a $40-50 \mathrm{~cm}$ interval of concentrated IRD, the stratigraphically lowest such occurrence at Site 748 (Fig. 5). The oxygen isotopic enrichment initiates at $117 \mathrm{mbsf}$ where the IRD first occur, and peaks at $115.5 \mathrm{mbsf}$ near the top of the IRD interval.

\section{Stable Carbon Isotopes}

Overall, Chiloguembelina consistently yielded the most positive carbon isotopic composition of all planktonic foraminifers analyzed, further suggesting that they maintained a depth habitat in the upper part of the photic zone. Subbotina angiporoides yielded values $0.4 \%$ o to $0.6 \%$ o lower than Chiloguembelina. The $\delta^{13} \mathrm{C}$ values of Cibicidoides and Gyroidina were roughly equivalent at all levels, and 0.5 to $1.0 \%$ lower than those of Chiloguembelina. The ostracodes yielded the lowest and most variable $\delta^{13} \mathrm{C}$ values of all components analyzed.

The Paleocene/lower Eocene portion of the carbon isotope record, although composed of few data, shows a large depletion of $2.5 \%$ ofrom 380 to 300 mbsf (Fig. 2) that is similar in magnitude to shifts recorded at other DSDP and ODP sites (Shackleton et al., 1984; Miller et al., 1987b). The planktonic and benthic foraminifer $\delta^{13} \mathrm{C}$ values remain somewhat constant above the lower Eocene, to the upper Eocene. In the lower Oligocene, benthic foraminifer $\delta^{13} \mathrm{C}$ values increased from $0.8 \%$ to $0.9 \%$ from 116 to $115.2 \mathrm{mbsf}$, whereas the planktonic foraminifers Chiloguembelina and $S$. angiporoides show a $<0.2 \%$ increase (Fig. 3). This event, which effectively reduced the planktonic to benthic $\delta^{13} \mathrm{C}$ gradient by $50 \%$, was followed by a gradual $1.0 \%$ decrease in planktonic and benthic foraminifer $\delta^{13} \mathrm{C}$ values over the remaining lower Oligocene (118-110 mbsf). Above $100 \mathrm{mbsf}$ benthic foraminifer $\delta^{13} \mathrm{C}$ values remained constant until $81 \mathrm{mbsf}$, just above the position of the lower/upper Oligocene boundary, where a second abrupt increase of $0.75 \%$ o was recorded.

\section{Strontium Isotopes}

Because few faunal datums are currently available for dating the Oligocene, strontium isotope stratigraphy inte- grated with magnetostratigraphy and stable isotope stratigraphy may provide the most accurate means of correlating Oligocene pelagic sequences (e.g., Miller et al., 1988). In addition, recent investigations have indicated that some of the Oligocene faunal datums may be diachronous from basin to basin or over latitude (e.g., Hess et al., 1989; Aubry, this volume). To develop a strontium isotope age model for Site 748 , strontium isotope analyses were performed on 15 samples of mixed planktonic foraminifers from middle Eocene to upper Oligocene sediments at Site 748 (Table 3).

The Site $7488^{87} \mathrm{Sr} /{ }^{86} \mathrm{Sr}$ record displays no variation in the middle to upper Eocene range, with a mean value of 0.70770 , but it does show a gradual $6 \times 10^{-4}$ increase in ${ }^{87} \mathrm{Sr}{ }^{86} \mathrm{Sr}$ ratios throughout the Oligocene (Fig. 3). The Eocene ${ }^{87} \mathrm{Sr}{ }^{86} \mathrm{Sr}$ values are similar to published values from other DSDP sites (Hess et al., 1986). The ${ }^{87} \mathrm{Sr} /{ }^{86} \mathrm{Sr}$ increase measured over the Oligocene at Site 748 is equal to that measured over the Oligocene at other DSDP sequences (e.g., Koepnick et al., 1988; Miller et al., 1988; Hess et al., 1989).

\section{Trace Elements and Fossil Preservation}

The concentration of certain trace elements in biogenic calcite changes during diagenesis (e.g., Baker et al., 1982; Veizer, 1978). The direction of change depends upon the distribution coefficient of the trace element and its availability in the diagenetic system or pore water. Because the distribution coefficients of these trace elements are roughly known (e.g., Lorens, 1981), one can often predict the direction and degree of change that will result from increased diagenesis. Thus, trace element ratios may be used to identify biogenic calcite that has been recrystallized or contaminated with diagenetic cements and overgrowths.

The concentrations of four major and minor trace elements ( $\mathrm{Sr}, \mathrm{Mg}, \mathrm{Fe}$, and $\mathrm{Mn}$ ) were measured in 22 mixed planktonic foraminifer samples from 60 to $120 \mathrm{mbsf}$. The data are reported in mole ratios to $\mathrm{Ca}(\mathrm{mM} / \mathrm{M})$ (Table 4$)$. The measured $\mathrm{Sr} / \mathrm{Ca}$ ratios range from 1.25 to $1.55(\mathrm{mM} / \mathrm{M}$ ) (Fig. 6). Although the value range is small, the $\mathrm{Sr} / \mathrm{Ca}$ ratios show a distinct change in the interval where the oxygen isotope shift was recorded, decreasing by $20 \%$ at $120 \mathrm{mbsf}$. The $\mathrm{Mg} / \mathrm{Ca}$ ratios show a slight peak over this interval as well, increasing by 0.5 from 127 to $120 \mathrm{mbsf}$ and decreasing by the same amount from 120 to $109 \mathrm{mbsf}$. Both $\mathrm{Fe} / \mathrm{Ca}$ and $\mathrm{Mn} / \mathrm{Ca}$ ratios increase slightly from the middle Eocene to the upper Oligocene. The mean values are slightly higher than those recorded for reductively cleaned core top planktonic foraminifers from the North Atlantic (e.g., Boyle, 1983), suggesting that Site 748 foraminifers are coated with Fe-Mn oxides or early diagenetic $\mathrm{MnCO}_{3}$.

Several lines of evidence indicate that the planktonic and benthic microfossils analyzed in this study have not been affected significantly by burial diagenesis. First, visual examination of foraminifers by light microscope revealed no cement overgrowths or infillings. Second, the $\mathrm{Sr} / \mathrm{Ca}$ ratios measured in Oligocene planktonic foraminifers are well within the range of $\mathrm{Sr} / \mathrm{Ca}$ ratios recorded in modern planktonic foraminifers and within the range of $\mathrm{Sr} / \mathrm{Ca}$ ratios recorded for fossil planktonic foraminifers from other Oligocene pelagic sequences (Bender et al., 1975; Hess et al., 1989; Graham et al., 1982). Although the $\mathrm{Sr} / \mathrm{Ca}$ ratios of middle to upper Eocene planktonic foraminifers from Site 748 are higher than observed elsewhere, it is unlikely that the higher ratios are an artifact of diagenesis; dissolution and abiotic precipitation should decrease, not increase, the $\mathrm{Sr} / \mathrm{Ca}$ ratios of microfossil calcite (e.g., Matter et al., 1975; Baker et al., 1982). Moreover, the $\mathrm{Sr}$ isotope ratios of both Eocene and Oligocene microfossils are identical to those 


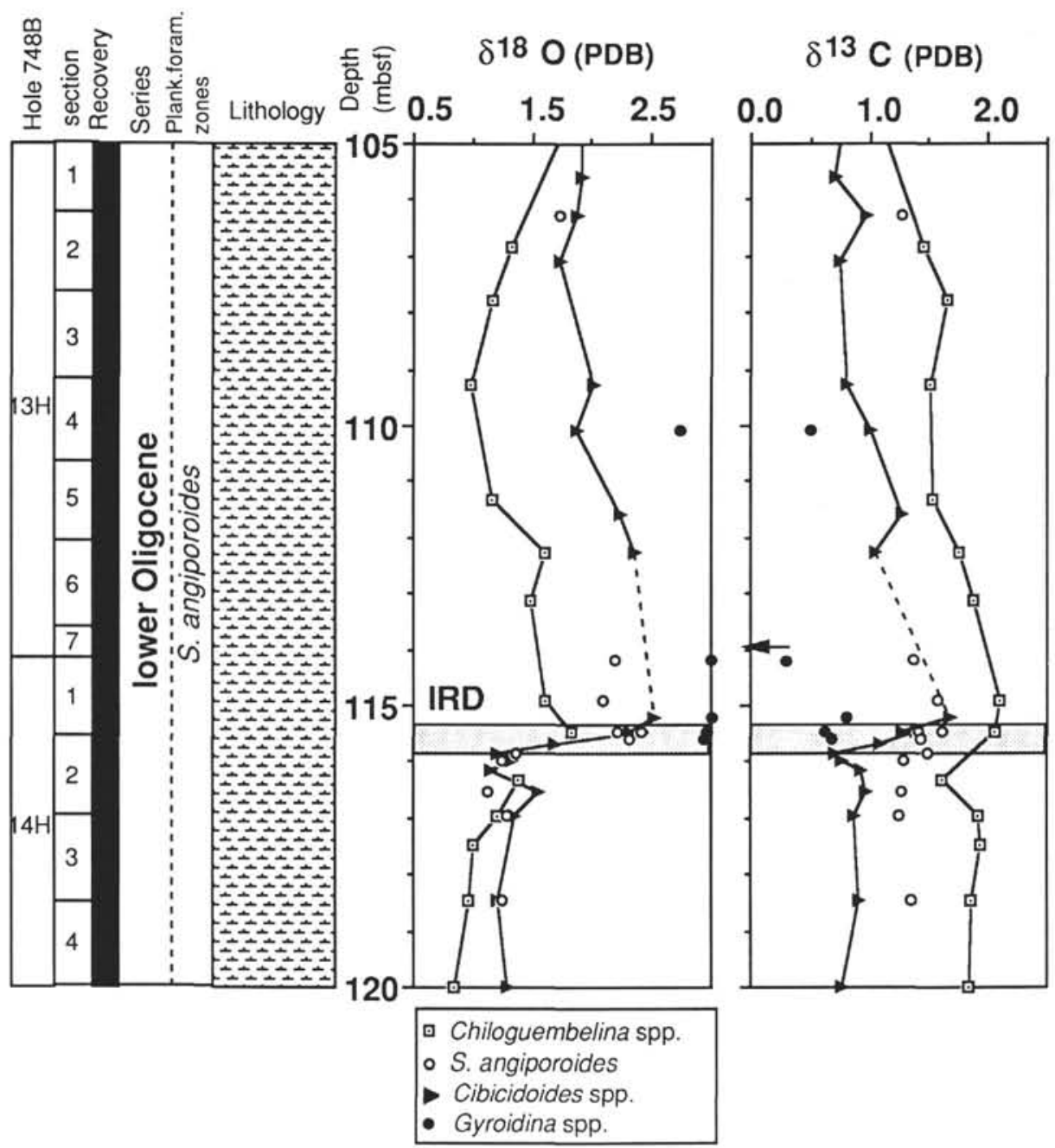

Figure 5. Expanded view of the oxygen and carbon isotope records for Site 748 over the earliest Oligocene IRD-bearing interval.

recorded at other pelagic sequences (Miller et al., 1988; Hess et al., 1989), which also indicates that $\mathrm{Sr}$ ions have not been remobilized. The early diagenetic carbonates should have a negligible effect on the isotopic composition of the foraminifers.

\section{Percent $\mathrm{CaCO}_{3}$}

Carbonate contents measured on whole-rock samples from the middle and upper Eocene at Site 748 yielded values with a mean carbonate content of $95 \%$ (Fig. 3). In the lower Oligocene, carbonate values decrease from $90 \%$ to $75 \%$ at 116 mbsf, reflecting the presence of IRD and glauconite and slightly higher concentrations of siliceous microfossils. From 115 to 105 mbsf, the carbonate contents remain low, varying between $70 \%$ and $85 \%$. Above $105 \mathrm{mbsf}$ the values increase to $90 \%$ and remain near this level until $75 \mathrm{mbsf}$, with the exception of a slight decrease at 90 mbsf. From 75 to 65 mbsf carbonate contents decrease from $90 \%$ to $70 \%$ as the concentration of siliceous microfossils increases.

\section{Chronology}

Site 748 age assignments for the Eocene and Oligocene are based primarily on faunal datums (Aubry, this volume; Berggren, this volume) and the $\mathrm{Sr}$ isotope stratigraphy (Table 6). Oligocene chronology is based on the LADs of Globigerin- atheka index (Eocene/Oligocene boundary), S. angiporoides, and Chiloguembelina (early/late Eocene boundary) at 120.5, 97.4, and 83.9 mbsf, respectively (Berggren et al., 1985). In addition, using the Site $748 \mathrm{Sr}$ isotope record and an Oligocene $\mathrm{Sr}$ isotope age equation calibrated to the geomagnetic polarity time scale (Miller et al., 1988), that is,

$$
t=20392.79-28758.84 \times{ }^{87} \mathrm{Sr} /{ }^{86} \mathrm{Sr}_{\text {sample }},
$$

the ages $26.1,31.4$, and $33.1 \mathrm{Ma}$ were assigned to three additional levels of the Oligocene, 67.0, 87.5, and $104.5 \mathrm{mbsf}$, respectively. The Eocene chronology is based on the LADs of Globigerinatheka, A. collectea, and A. primitiva. Ages for samples below the $A$. primitiva interval were extrapolated assuming constant sedimentation rates across the $A$. primitiva LAD. Because the Site 748 magneto- and biostratigraphic records may undergo further revision, the age estimates presented here should be considered as preliminary.

The Sr-isotope-derived ages for two samples from 87.5 and $104.5 \mathrm{mbsf}$ in the lower and upper early Oligocene appear to be consistent with faunal LADs (Table 6). The Sr-isotope-derived age for the sample at $67 \mathrm{mbsf}$, however, is $26.1 \mathrm{Ma}$, whereas the published age of the nearest faunal LAD ( $G$. euapertura) at 66.0-66.7 mbsf is $23.5 \mathrm{Ma}$. This would suggest that either the $G$. euapertura $\mathrm{LAD}$ is diachronous or the $\mathrm{Sr}$ isotope equation or 


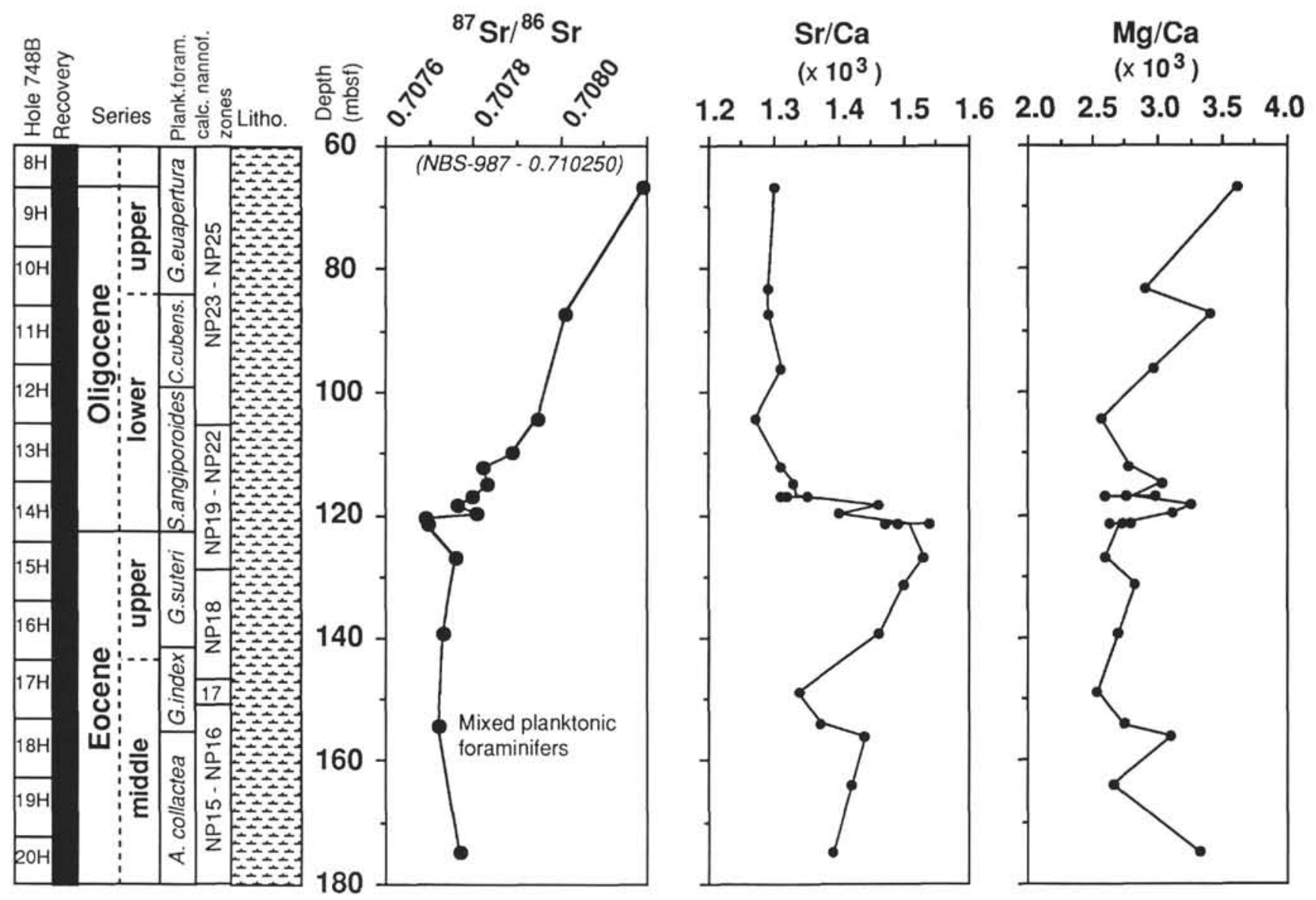

Figure $6 .{ }^{87} \mathrm{Sr} /{ }^{86} \mathrm{Sr}$ isotope and $\mathrm{Sr} / \mathrm{Ca}$ and $\mathrm{Mg} / \mathrm{Ca}$ molar ratios of the mixed planktonic foraminifer samples plotted vs. depth, Site 748 .

Table 6. Depths and ages of faunal, floral, and ${ }^{87} \mathrm{Sr} /{ }^{86} \mathrm{Sr}$ criteria used to establish the chronology of Site 748 and to calculate the sedimentation rates.

\begin{tabular}{lrr}
\hline \multicolumn{1}{c}{ Criterion } & $\begin{array}{r}\text { Depth } \\
\text { (mbsf) }\end{array}$ & $\begin{array}{c}\text { Age } \\
\text { (Ma) }\end{array}$ \\
\hline Globigerina euapertura & 66.60 & 24.0 \\
Sr isotopes & 67.00 & 26.1 \\
Chiloguembelina cubensis LAD & 83.90 & 30.0 \\
Sr isotopes & 87.50 & 31.4 \\
Subbotina angiporoides LAD & 97.40 & 32.0 \\
Sr isotopes & 104.50 & 33.1 \\
Reticulofenstra hillae LAD & 106.00 & 34.6 \\
Globigerinatheka index LAD & 120.50 & 36.7 \\
Isthmolithus recurvus FAD & 128.00 & 37.8 \\
Acarinina collactea & 142.00 & 40.6 \\
Chiasmolithus oamaruensis FAD & 146.50 & 39.8 \\
Acarinina primitiva LAD & 152.50 & 42.8 \\
\hline & & \\
\hline
\end{tabular}

Notes: Age estimates of datum events are from Berggren et al., $1985 ;{ }^{87} \mathrm{Sr} /{ }^{86} \mathrm{Sr}$ based age estimates were calculated by means of the $\mathrm{Sr}$ isotope age model of Miller et al., 1988.

value is in error. Because recent isotope studies have demonstrated substantial diachrony for some commonly used Oligocene faunal zones (Hess et al., 1989; Aubry, this volume), we have chosen to use the $\mathrm{Sr}$-isotope-derived age estimate for the uppermost Oligocene in our age model.

Using the above chronology, the Site 748 foraminifer stable isotope values have been plotted vs. a numerical age frame- work (25-45 Ma) in Figure 7. For comparison, the benthic foraminifer oxygen isotope values, as well as published data from five other DSDP and ODP sites, have also been plotted vs. time in Figure 9. The oxygen isotope trends recorded at Site 748 from middle Eocene to middle Oligocene appear to correlate well with the trends documented at other sites indicating that the chronology used here is consistent with those for other sequences. One event not observed at Site 748 is the ubiquitous middle Oligocene $\delta^{18} \mathrm{O}$ increase at $30 \mathrm{Ma}$ (e.g., Miller et al., 1987a; Kennett and Stott, 1990), indicating the possible presence of a 1-2 m.y. hiatus at this level.

\section{DISCUSSION}

The unique feature of the Site 748 Eocene-Oligocene sequence is the presence of IRD within the same interval where the ubiquitous early Oligocene $\delta^{18} \mathrm{O}$ event occurs. Although early Oligocene glacial debris has been documented in deep-sea deposits in several other locations near the Antarctic continent, this is the first location where IRD has been directly linked with the early Oligocene $\delta^{18} \mathrm{O}$ event. The benthic foraminifer $\delta^{18} \mathrm{O}$ values at Site 748 significantly increase at the base of the IRD interval and attain a maximum near the center of this interval (Fig. 7). Above the IRD, benthic foraminifer $\delta^{18} \mathrm{O}$ values decrease but do not reach values as low as those below the IRD. The mineralogy of the IRD, angular quartz, mica, and heavy minerals, is similar to debris found in Prydz Bay during Leg 119; therefore, it is probable that the source of the debris was the Antarctic continent (Breza and Wise, this volume). Considering the distance between Prydz Bay and Site $748(\sim 1200 \mathrm{~km})$, the 


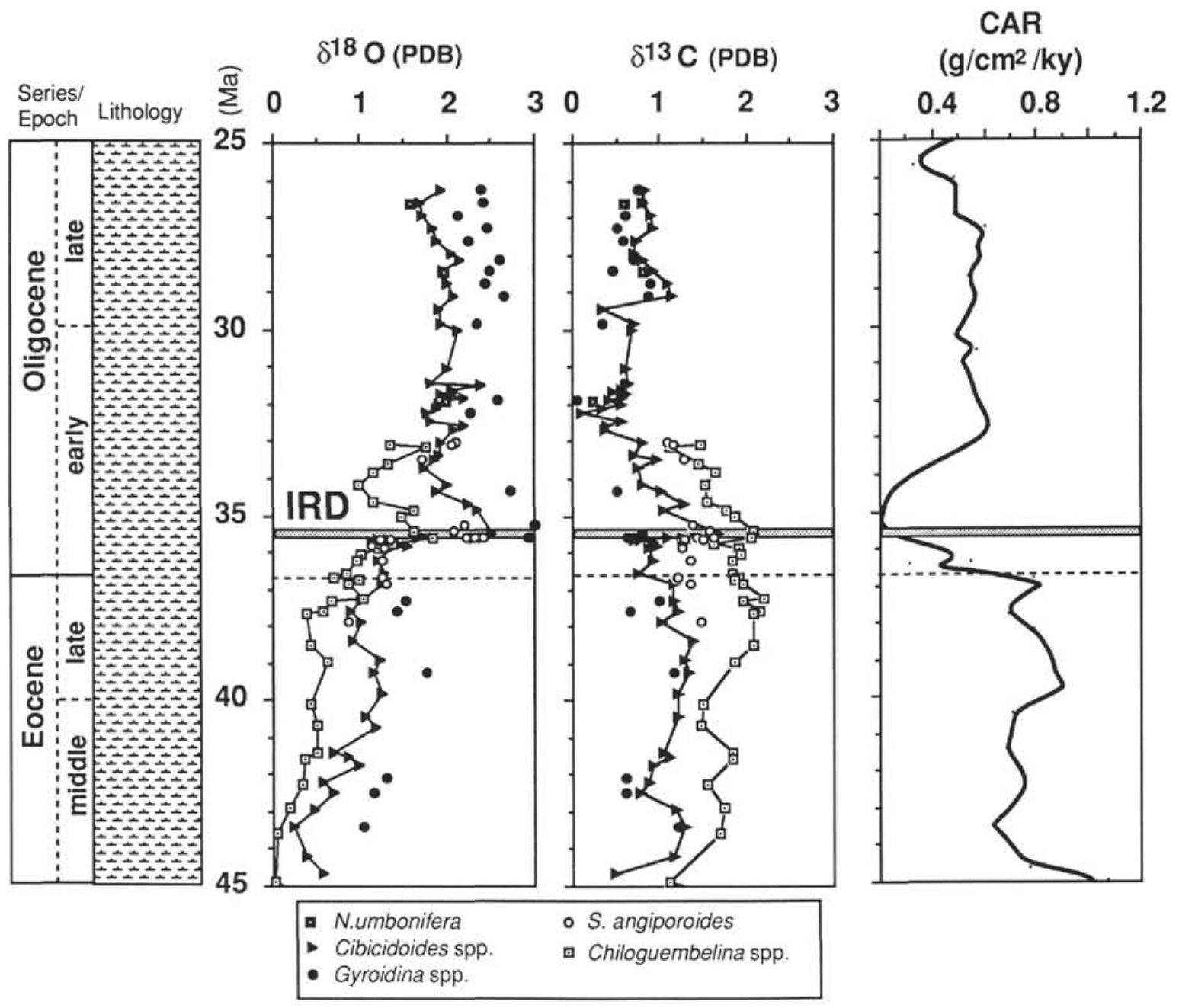

Figure 7. Stable oxygen and carbon isotope data and carbonate accumulation rates (CAR) plotted vs. age, Site 748 . The Eocene and Oligocene ages are based on the $\mathrm{Sr}$ isotope stratigraphy, planktonic foraminifer and calcareous nannofossil zonations, Miller et al.'s (1988) Sr isotope age model, and the Berggren et al. (1985) time scale (Table 6). CARs were calculated by means of the following equation: $\mathrm{CAR}=\% \mathrm{CaCO}_{3} / 100 \times \mathrm{DBD} \mathrm{g} / \mathrm{cm}^{3} \times \mathrm{S} \mathrm{cm} / \mathrm{yr}, \% \mathrm{CaCO}_{3}$ values, and physical properties measurements of dry-bulk density (DBD) (Part A).

only viable mechanism for transporting the larger grains found at Site 748 between these two locations was via icebergs. Thus, the sudden occurrence of IRD in the lower Oligocene at Site 748 provides direct evidence that icebergs, originating on the margin of the Antarctic continent, were passing over the Kerguelen Plateau at the time the ubiquitous $\delta^{18} \mathrm{O}$ shift was recorded by calcareous microfossils.

Tillite and other IRD in Paleogene terrestrial and marine sequences discovered near Antarctica provide direct evidence that glacial activity has occurred on the continent since the middle Eocene (e.g., Margolis and Kennett, 1971; Hayes, Frakes, et al., 1975; Birkenmajer et al., 1989; Barrett et al., 1989). IRD, however, is a common product of both alpine glaciers and continental ice sheets; thus, the relationship of these Paleogene IRD deposits to global ice volume is uncertain (e.g., Kennett and Barker, 1990). This uncertainty applies to the IRD found at Site 748 as well. Although the presence of IRD at Site 748 establishes that glacial activity increased simultaneously with the early Oligocene oxygen isotope event, it alone does not unequivocally prove that the $\delta^{18} \mathrm{O}$ increase was brought about by increased global ice volume.

At present, the only adequate means of quantitatively assessing global ice-volume changes is through the marine $\delta^{18} \mathrm{O}$ record as measured in biogenic calcite. This method is based on the observation that water evaporated from the oceans and precipitated in high latitudes is isotopically depleted relative to seawater. Because ice sheets contain large quantities of this water, past variations in the size of continental ice sheets have produced measurable changes in the $\delta^{18} \mathrm{O}$ composition of the ocean, a record of which is preserved in calcareous microfossils. The oxygen isotope method, however, is complicated by the fact that $\delta^{18} \mathrm{O}$ ratios of calcareous fossils are also influenced by the temperature of water in which they were formed. As a result, it is difficult to separate the relative effects of ice volume and temperature on microfossil $\delta^{18} \mathrm{O}$ values.

This specific problem has been the central issue in the debate over the origin of the early Oligocene $\delta^{18} \mathrm{O}$ enrichment. Citing the paucity of large scale deposits of Oligocene glacial debris off the margins of Antarctica, some researchers have argued that the $1.2 \%$ enrichment recorded by early Oligocene benthic foraminifers represents a $>5^{\circ} \mathrm{C}$ cooling of deep-ocean waters without substantial change in ice volume (Shackleton and Kennett, 1975). In contrast, others have maintained that the $\delta^{18} \mathrm{O}$ shift represents mainly the growth of ice sheets on the Antarctic continent, because $\delta^{18} \mathrm{O}$ increases of a similar mag- 
nitude were recorded by planktonic foraminifers from western equatorial regions, where sea-surface temperatures (SST) are thought to have been constant throughout time (e.g., Matthews and Poore, 1980; Prentice and Matthews, 1988). In fact, using the assumption of constant SST in the western tropics, Prentice and Matthews (1988) have theorized that a permanent ice sheet has been present on East Antarctica since the early Eocene. In addition, Miller et al. (1987a) noted that benthic foraminifer values greater than $1.8 \%$ in an ice-free world would require deep-water temperatures colder than at present and suggested that the East Antarctic ice sheet only existed during those intervals of time in which benthic foraminifer $\delta^{18} \mathrm{O}$ values were greater than $1.8 \%$.

A potentially more efficient approach for estimating the contribution of ice-volume changes to the early Oligocene $\delta^{18} \mathrm{O}$ event involves reconstruction and comparison of planetary $\delta^{18} \mathrm{O}$ gradients for intervals of time bounding that event. If one assumes that late Eocene and Oligocene decreases in deep-water temperatures were roughly paralleled by equal decreases in high-latitude, surface-water temperatures while tropical temperatures remained constant or changed by a small amount (e.g., Adams et al., 1990), then it follows that an early Oligocene decrease in high-latitude temperatures should have been accompanied by an increase in planetary temperature gradients. But if the $\delta^{18} \mathrm{O}$ increase was due solely to an ice-volume increase, then the planetary $\delta^{18} \mathrm{O}$ gradients should have remained unchanged as absolute values increased in unison everywhere.

Keigwin and Corliss (1986) first attempted to estimate the late Eocene to early Oligocene planetary temperature gradients by reconstructing latitudinal surface water $\delta^{18} \mathrm{O}$ gradients for broad intervals of time preceding and following the $\delta^{18} \mathrm{O}$ shift. They plotted average $\delta^{18} \mathrm{O}$ values of late Eocene and early Oligocene surface-dwelling planktonic foraminifers from many DSDP sites vs. "absolute" paleolatitude corrected for past plate motions. Their reconstructions implied that planetary $\delta^{18} \mathrm{O}$ gradients did not change significantly from the late Eocene to the early Oligocene. The accuracy of those latitudinal temperature gradients, however, was compromised by the lack of data from high-latitude regions. To address this deficiency, we modified the Keigwin and Corliss (1986) plots for the late Eocene and early Oligocene by adding planktonic foraminifer $\delta^{18} \mathrm{O}$ data from Site 748 as well as data from another high-latitude site located on Maud Rise, Site 689 (Stott et al., 1990) (Fig. 8A). In addition, an early Eocene gradient was constructed for comparison (Fig. 8B). The most negative planktonic foraminifer $\delta^{18} \mathrm{O}$ values were chosen from each site, assuming these values most closely represented upper mixed layer conditions (e.g., Poore and Matthews, 1984). The late Eocene and early Oligocene $\delta^{18} \mathrm{O}$ data were fit to polynomial (quadratic) regression curves.

With the addition of new high-latitude data to the Keigwin and Corliss (1986) data base it becomes apparent that late Eocene and early Oligocene high-latitude temperatures were much lower than estimated in the original reconstruction. The late Eocene and early Oligocene planktonic foraminifer $\delta^{18} \mathrm{O}$ values from Sites 748 and 689 are as much as $0.75 \%$ to $1.0 \%$ o higher than the values predicted from the previous gradient. The revised early Oligocene $\delta^{18} \mathrm{O}$ gradient shows a slightly greater slope than the late Eocene gradient toward high latitudes (Fig. 8A). The mean departure in latitudinal $\delta^{18} \mathrm{O}$ gradients from the late Eocene to early Oligocene at low latitudes is about $0.3 \%$ to $0.4 \%$. This offset, assuming constant tropical SST, represents the portion of the early Oligocene $\delta^{18} \mathrm{O}$ increase that may be attributable to ice-volume change (Keigwin and Corliss, 1986). The departure between the curves at $70^{\circ}$ latitude is $0.6 \%$, suggesting that $0.2 \%$ to
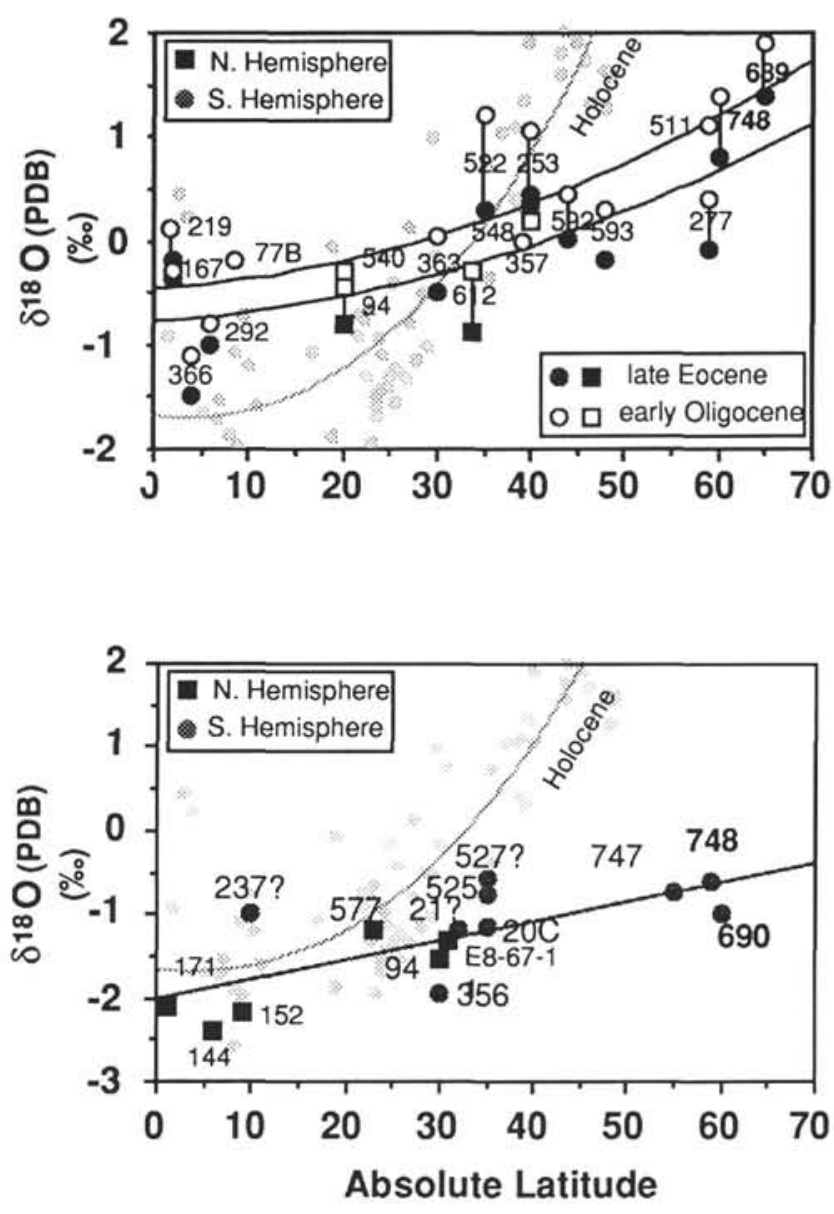

Figure 8. Latitudinal surface-water $\delta^{18} \mathrm{O}$ gradients of the (A) late Eocene and early Oligocene and (B) early Eocene. Also plotted are the $\delta^{18} \mathrm{O}$ values of surface-dwelling planktonic foraminifers from various sites vs. the absolute latitude of the site corrected for past plate motions. The late Eocene and early Oligocene data were originally compiled by Keigwin and Corliss (1986). We have added data from high-latitude Sites 689 (Stott et al., 1990) and 748. The paleolatitude of Site 748 at $35 \mathrm{Ma}$ was estimated to be $60^{\circ} \mathrm{S}$ based on reconstructions of Mutter and Cande (1983). The late Eocene and early Oligocene data were fitted with quadratic regression curves $(R 2$ $=0.65$ and 0.60 ). Isotope data for the early Eocene reconstruction are from Douglas and Savin (1973), Shackleton and Kennett (1975), Shackleton and Boersma (1981), Oberhänsli et al. (1984, 1986), Shackleton et al. (1984, 1985), Boersma et al. (1987), and Stott et al. (1990). The early Eocene data were fit to a linear regression line $\left(R^{2}=\right.$ 0.53 ) because attempts to fit the data to nonlinear regression curves produced poor correlation coefficients. The shaded background points and curve in each plot represent the Holocene (core tops), planktonic foraminifer $\delta^{18} \mathrm{O}$ gradient for the Southern Hemisphere (after Savin et al., 1985).

$0.3 \%$ of the earliest Oligocene $\delta^{18} \mathrm{O}$ increase at high latitude was caused by lowered temperatures. Similarly, the $1.0 \%$ $1.2 \%$ increase recorded by benthic foraminifers in the earliest Oligocene probably represents the combined affects of a $3^{\circ}-4^{\circ} \mathrm{C}$ decrease in deep-water temperatures and a $0.4 \%$ o ice-volume signal (e.g., Miller et al., 1987a).

The earliest Oligocene decrease in high-latitude temperatures, however, was small in comparison with the longer term trend recorded earlier in the Eocene. From the early to late Eocene high-latitude planktonic foraminifer $\delta^{18} \mathrm{O}$ values in- 
creased by as much as $1.75 \%$, whereas low-latitude values increased by less than $0.5 \%$ (Fig. $8 \mathrm{~B}$ ). Benthic foraminifer $\delta^{18} \mathrm{O}$ values increased by $1.5 \%$ over the same period (Miller et al., 1987a). The equivalent early to late Eocene increases in benthic and high-latitude planktonic foraminifer $\delta^{18} \mathrm{O}$ values indicate that much of the increase was brought about by high-latitude and deep-ocean cooling.

A combined ice-volume and temperature change as the origin of the early Oligocene benthic foraminifer $\delta^{18} \mathrm{O}$ increase helps explain observed changes in the vertical $\delta^{18} \mathrm{O}$ gradient over the Kerguelen Plateau (Fig. 5). In the upper Eocene at Site 748, Chiloguembelina, S. angiporoides, and Cibicidoides show very similar $\delta^{18} \mathrm{O}$ values indicating that the water column over Kerguelen Plateau possessed a small thermal gradient. During the early Oligocene, however, Chiloguembelina increased by only $0.5 \%$, whereas $S$. angiporoides increased by $1.0 \%$ and Cibicidoides by $1.2 \%$; thus, the vertical $\delta^{18} \mathrm{O}$ gradient expanded. The larger increase for Cibicidoides reflects the added affect of $3^{\circ}-4^{\circ} \mathrm{C}$ cooler bottom waters in the Oligocene, whereas the shift recorded by Chiloguembelina mainly reflects ice volume. $S$. angiporoides, an inferred deeper dwelling planktonic foraminifer, also recorded a temperature decrease but to a smaller degree because it resided at intermediate depths within the thermocline.

A smaller early Oligocene temperature decrease is also more compatible with the record of faunal and flora turnover. Globally, it appears that much of the succession of warm water taxa by cold water taxa occurred before the early Oligocene. Transition events for planktonic and benthic foraminifer, calcareous nannoplankton, and deep-sea ostracode assemblages were recorded in several distinct intervals during the middle or late Eocene, but not in the early Oligocene (Benson, 1975; Corliss, 1979; Aubry, 1983; Keller, 1983a, 1983b; Benson et al., 1984; Snyder et al., 1984; Corliss and Keigwin; 1986; McGowan and Beecroft, 1986; Boersma et al., 1987; Thomas, 1990). Also, planktonic foraminifer depth stratification, an important indicator of vertical temperature gradients in the surface ocean, underwent dramatic changes in the middle and late Eocene, prior to the early Oligocene event (Keller, 1983a; Boersma et al., 1987). Investigations of the microfauna and microflora assemblages from Site 748 cores have revealed a similar pattern. Aubry (this volume) shows that there was a major reduction in diversity of the calcareous nannoplankton at Site 748 near the early/middle Eocene boundary, but there were no changes associated with the Eocene/Oligocene boundary. Results of a principal component analysis show that the major turnover in benthic foraminifer populations on the Kerguelen Plateau occurred in the late middle Eocene (Mackensen and Berggren, this volume).

The Site 748 Eocene/oligocene benthic foraminifer isotope record shows several distinct features that have been observed at other pelagic sequences, including the $1.2 \% 0 \delta^{18} \mathrm{O}$ increase in the lower Oligocene, a second $0.5 \% \circ \delta^{18} \mathrm{O}$ increase in the middle Oligocene, a gradual $\delta^{18} \mathrm{O}$ decrease in the late Oligocene, and a gradual decline in $\delta^{13} \mathrm{C}$ values during the early Oligocene (see Miller et al., 1987a, 1989). One interesting feature of the Site 748 record that was not observed at every site is a rapid increase in benthic foraminifer $\delta^{13} \mathrm{C}$ values that corresponds with the early Oligocene $\delta^{18} \mathrm{O}$ increase. Similar shifts in benthic foraminifer $\delta^{13} \mathrm{C}$ values have been recorded for the same interval at Sites 522 and 563 in the Atlantic and 574 in the Pacific (Miller et al., 1985; Miller and Thomas, 1985; Miller et al., 1988). The $\delta^{13} \mathrm{C}$ increase is not recorded in planktonic foraminifers at this location, and thus may reflect a change in the chemistry or sources of deep and intermediate depth waters. A shift to higher $\delta^{13} \mathrm{C}$ values would imply a transition to younger, more nutrient-depleted waters.

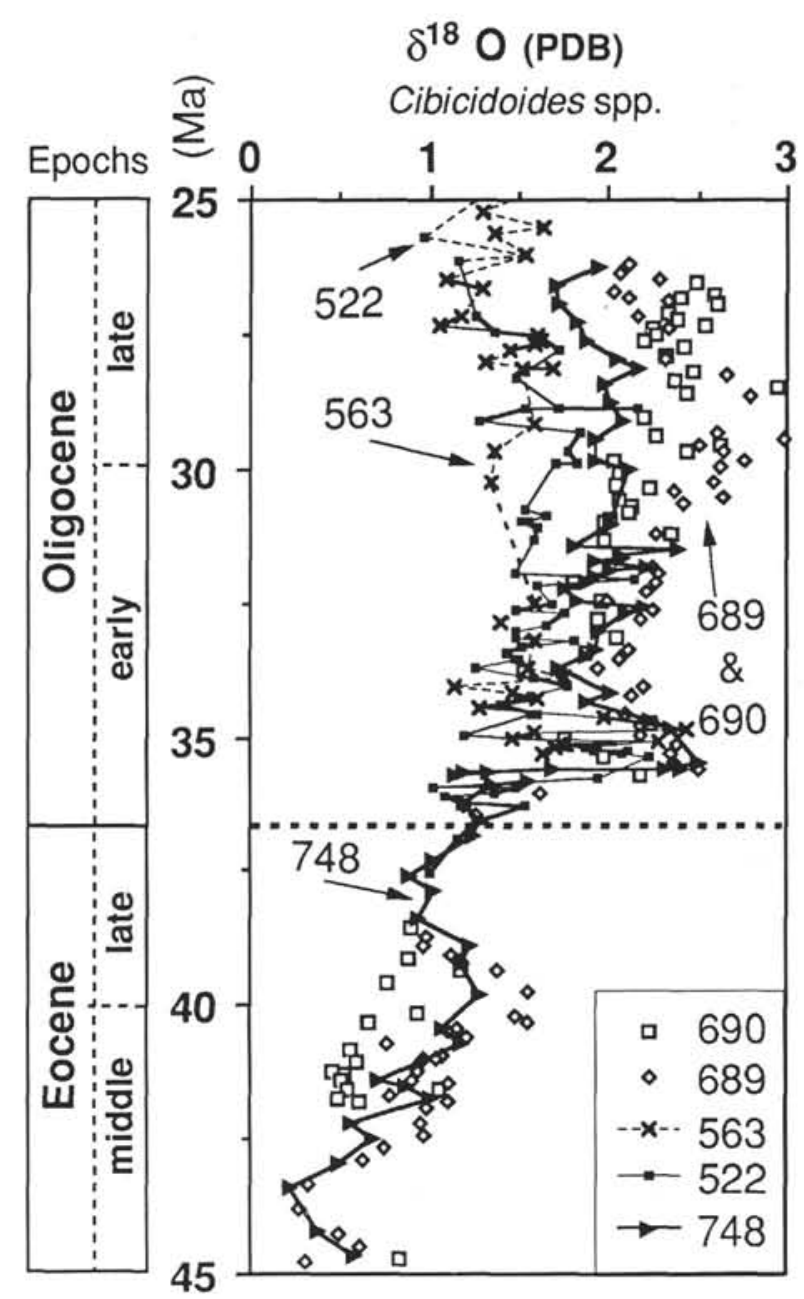

Figure 9. Compilation of late Eocene and Oligocene Cibicidoides oxygen isotope data from Site 748; Sites 522 and 563 located in the South and North Atlantic, respectively (Miller et al., 1985, 1988); and Sites 689 and 690 located on Maud Rise in the Southern Ocean (Kennett and Stott, 1990). Published chronologies for each site are based on the Berggren et al. (1985) time scale.

This may occur if the deep-water source to a specific region became more proximal. Such might have been the case during the early Oligocene for the Kerguelen Plateau and the surrounding southern Indian ocean. The rapid transition to slightly colder and more nutrient-depleted intermediate waters in the early Oligocene would implicate the Antarctic margin as this most proximate source.

Another unique feature of the Site 748 benthic foraminifer record is revealed by a compilation of benthic foraminifer $\delta^{18} \mathrm{O}$ values. Figure 9 shows Cibicidoides data from Site 748 and published benthic foraminifer $\delta^{18} \mathrm{O}$ data from four other sites plotted against age. Sites 522 and 563 were drilled in the South and North Atlantic and had early Oligocene paleodepths of 2693 and $2386 \mathrm{~m}$, respectively (Miller et al., 1985, 1987a). Sites 689 and 690, drilled on the Maud Rise in the Southern Ocean, had early Oligocene paleodepths of 1500 and $2350 \mathrm{~m}$ (Kennett and Stott, 1990). Following the early Oligocene increase, the Site 748 benthic foraminifer $\delta^{18} \mathrm{O}$ values did not decrease significantly but remained high, near $2.0 \%$, for the remainder of the Oligocene. This is in sharp contrast to the benthic foraminifer $\delta^{18} \mathrm{O}$ records of Atlantic Sites 522 and 563, which show a return to a mean value of $1.5 \%$ by the middle early 
Oligocene, and which only exceeded $1.6 \%$ for a short interval in the middle Oligocene (Fig. 9). Sites 689 and 690, similar to Site 748 , show high $\delta^{18} \mathrm{O}$ values through the Oligocene. Thus, new data from the Southern Ocean sites significantly increases the range of benthic $\delta^{18} \mathrm{O}$ values observed for the Oligocene (Fig. 9).

This phenomenon has several important implications. First, the higher benthic foraminifer $\delta^{18} \mathrm{O}$ values recorded at high-latitude sites indicate that the oceans became more thermally segregated in the Oligocene. Murphy and Kennett (1986) documented a similar Oligocene separation of benthic and planktonic foraminifer $\delta^{18} \mathrm{O}$ values in the southwest $\mathrm{Pa}$ cific and suggested that this separation marked the establishment of a distinct thermal segregation of surface-water masses in the southwest Pacific that resulted from intensification of the circum-Antarctic current. A second implication of the higher Oligocene benthic foraminifer $\delta^{18} \mathrm{O}$ values involves the debate over ice volume and permanent ice sheets. It has been suggested that benthic $\delta^{18} \mathrm{O}$ values greater than $1.8 \%$, assuming an ice-free world, yield bottom-water temperatures cooler than present-day temperatures in many locations, so that large ice sheets must have existed during intervals of time represented by values exceeding $1.8 \%$ (Miller et al., 1987). Before recent high-latitude drilling by ODP, however, there were only two intervals of the Oligocene in which benthic foraminifer $\delta^{18} \mathrm{O}$ values exceeded $1.8 \%$, the earliest and middle Oligocene. Because the benthic foraminifer $\delta^{18} \mathrm{O}$ values from Site 748 as well as from Sites 689 and 690 on Maud Rise (Kennett and Stott, 1990) remain well above this value throughout the Oligocene (Fig. 8), following the above argument, one would have to assume an ice sheet was present on the Antarctic continent during the entire Oligocene. Alternatively, the higher benthic foraminifer $\delta^{18} \mathrm{O}$ values recorded at Kerguelen Plateau and Maud Rise may reflect higher salinity values rather than colder temperatures.

Although the relative timing and magnitude of the oxygen isotopic events and the IRD at Site 748 provide further support for an ice-volume increase as the source of the early Oligocene $\delta^{18} \mathrm{O}$ shift, a suitable triggering mechanism for this event is still not apparent. The cooling of the high latitudes during the Eocene appears to be a long-term event that has been attributed to the gradual thermal isolation of the Antarctica continent because of the widening of the oceanic passages between Antarctica and South America and Australia (e.g., Kennett and Shackleton, 1976; Kennett, 1977). Thus, it is unclear why significant ice accumulation would suddenly initiate without major cooling. One possible explanation is that ice accumulation on Antarctica began when the mean annual temperatures at the southern high latitudes fell below a certain threshold level, after which the Antarctic summers were too cold to melt the snow completely from the previous winter (e.g., Shackleton and Kennett, 1975; Crowley et al., 1988). Such rapid changes in ice-sheet growth dynamics may be a normal response to gradual forcing functions. Computer modeling experiments have demonstrated that the growth and size of polar ice caps may largely be controlled by the heat sink distribution along an ice sheet's edge, which in turn is a function of ice-sheet size (North, 1984; Crowley and North, 1990). Ice sheets with a radius smaller than a certain critical size were found to be unstable and incapable of sustaining growth, whereas larger ice sheets were more stable and could expand rapidly. In the case of a polar continent experiencing gradual cooling as a result of tectonic isolation, such as Antarctica during the Eocene and Oligocene, large ice sheets may not have appeared until a critical temperature and/or ice-sheet size was surpassed. Alternatively, past changes in ice-sheet size may have simply been controlled by the amount of precipitation reaching a continent, which in turn might have been a function of surrounding ocean temperatures (i.e., the "snow-gun" hypothesis; Schnitker, 1980; Prentice and Matthews, 1988). Although such models and hypotheses still require further testing, they demonstrate that potentially rapid changes in global ice volume need not be accompanied by large, rapid changes in planetary temperature gradients.

\section{CONCLUSIONS}

The planktonic foraminifer, calcareous nannofossil, and ${ }^{87} \mathrm{Sr} /{ }^{86} \mathrm{Sr}$ and stable isotope stratigraphies indicate that the middle Eocene to upper Oligocene sequence at Site 748 is complete, with the exception of a possible 1-2 m.y. hiatus in the middle Oligocene. Thus, Site 748 provides a continuous record of the paleoenvironmental changes in the southern Indian Ocean during the Eocene-Oligocene transition. Based on Site 748 planktonic and benthic foraminifer oxygen isotope records and records from other high-latitude sites, it appears that much of the Paleogene high-latitude and deep-ocean cooling occurred in the middle to late Eocene, with $3^{\circ}-4^{\circ} \mathrm{C}$ additional cooling during the Eocene/Oligocene boundary event. Furthermore, the coeval occurrence of IRD and a $>1.0 \% 0 \delta^{18} \mathrm{O}$ shift in benthic foraminifers provide the first direct link of glacial activity on the Antarctic continent and the ubiquitous early Oligocene oxygen isotope event. Reconstructions of latitudinal $\delta^{18} \mathrm{O}$ gradients indicate that $0.3 \% 0-0.4 \%$ of the early Oligocene increase can be attributed to ice-volume effects. Finally, the benthic foraminifer $\delta^{18} \mathrm{O}$ and $\delta^{13} \mathrm{C}$ records suggest that, during the earliest Oligocene, the Antarctic became the primary source of intermediate depth waters to the southern Indian Ocean.

\section{ACKNOWLEDGMENTS}

This research was supported by JOIDES-USSAC Grant No. 20238. We are indebted to Dr. Ted Huston, Jason Curtis, and Scott Carpenter for technical assistance. Dr. Norm Macleod assisted with microfossil identification. We would also like to thank Drs. K. Miller, L. Stott, L. Sloan, K. C. Lohmann, T. Quinn, D. Rea, and J. Walker for their comments and stimulating discussion. Drs. James P. Kennett, Heidi Oberhänsli, and Ellen Thomas supplied critical reviews that helped improve the manuscript.

\section{REFERENCES}

Adams, G. C., Lee, D. E., and Rosen, B. R., 1990. Conflicting isotopic and biotic evidence for tropical sea-surface temperatures during the Tertiary. Palaeogeogr., Palaeoclimatol., Palaeoecol., 77:289-313.

Aubry, M.-P., 1983. Late Eocene to early Oligocene calcareous nannoplankton biostratigraphy and biogeography. AAPG Bull., $67: 415$.

Axelrod, D. I., 1984. An interpretation of Cretaceous and Tertiary biota in polar regions. Palaeogeogr., Palaeoclimatol., Palaeoecol., 45:105-147.

Baker, P. A., Gieskes, J. M., and Elderfield, H., 1982. Diagenesis of carbonates in deep-sea sediments: evidence from $\mathrm{Sr}^{2+} / \mathrm{Ca}^{2+}$ ratios and interstitial dissolved $\mathrm{Sr}^{2+}$ data. J. Sediment. Petrol., 52:71-82.

Barrett, P. J., Hambrey, M. J., Harwood, D. M., Pyne, A. R., and Webb, P.-N., 1989. Synthesis. In Barrett, P. J. (Ed.), Antarctic Cenozoic History from the CIROS-1 Drillhole, McMurdo Sound. DSIR Bull., 245:241-251.

Barron, E. J., 1987. Eocene equator-to-pole surface ocean temperatures: a significant climate problem? Paleoceanography, 2:729739.

Bender, M. L., Lorens, R. B., and Williams, D. F., 1975. Sodium, magnesium, and strontium in the tests of planktonic foraminifera. Micropaleontology, 21:448-459.

Benson, R. H., 1975. The origin of the psychrosphere as recorded in changes of deep sea ostracode assemblages. Lethaia, 8:69-83. 
Benson, R. H., Chapman, R. E., and Deck, L. T., 1984. Paleoceanographic events and deep-sea ostracodes. Science, 224:1334-1336.

Berggren, W. A., Kent, D. V., Flynn, J. J., and Van Couvering, J. A., 1985. Cenozoic geochronology. Geol. Soc. Am. Bull., 96:14071418.

Birkenmajer, K., Delitata, M. C., Narebski, W., Nicoletti, M., and Petrucciani, C., 1986. Geochronology of Tertiary island-arc volcanics and glacigenic deposits, King George Island, South Shetland Islands (West Antarctica). Bull. Pol. Acad. Sci. Earth Sci., 34:257-273.

Boersma, A., Premoli Silva, I., and Shackleton, N. J., 1987. Atlantic Eocene planktonic foraminiferal paleohydrographic indicators and stable isotope paleoceanography. Paleoceanography, 2:287-331.

Boyle, E. A., 1983. Manganese carbonate overgrowths on foraminifera tests. Geochim. Cosmochim. Acta, 47:1815-1819.

Brass, G. W., Southam, J. R., and Peterson, W. H., 1982. Warm saline bottom water in the ancient ocean. Nature, 296:620-623.

Carpenter, S. J., Lohmann, K. C, Holden, P., Walter, L. M., Huston, T., and Halliday, A. N., in press. $\delta^{18} \mathrm{O}$ values, $87 \mathrm{Sr} /{ }^{86} \mathrm{Sr}$ and $\mathrm{Sr} / \mathrm{Mg}$ ratios of late Devonian abiotic marine calcite: implications for the composition of ancient seawater. Geochim. Cosmochim. Acta.

Chamberlin, T. C., 1906. On a possible reversal of deep-sea circulation and its influence on geologic climates. J. Geol., 14:363-373.

Corliss, B. H., 1979. Response of deep-sea benthonic foraminifera to development of the psychrosphere near the Eocene/Oligocene boundary. Nature, 282:63-65.

Corliss, B. H., and Keigwin, L. D., 1986. Eocene/Oligocene paleoceanography. In Hsü, K. J. (Ed.), Mesozoic and Cenozoic Oceans: Am. Geophys. Union Geodyn. Ser., 15:101-118.

Craig, H., 1957. Isotopic standards for carbon and oxygen correction factors for mass spectrometric analysis of $\mathrm{CO}_{2}$. Geochim. Cosmochim. Acta, 12:133-149.

Crowley, T. J., and North, G. R., 1990. Modeling onset of glaciation. Ann. Glaciol., 14:39-42.

Crowley, T. J., Short, D. A., Mengel, J. G., and North, G. R., 1986. Role of seasonality in the evolution of climate over the last 100 million years. Science, 231:579-584.

Douglas, R. G., and Savin, S. M., 1973. Carbon and oxygen isotope analyses of Cretaceous and Tertiary foraminifer from central north Pacific. In Winterer, E. L., Ewing, J. I., et. al., Init. Repts. DSDP, 17: Washington (U.S. Govt. Printing Office), 591-606. 1975. Oxygen and carbon isotope analyses of Tertiary and Cretaceous microfossils from Shatsky Rise and other sites in the North Pacific Ocean. In Larson, R. L., Moberly, R., et al., Init. Repts. DSDP, 32: Washington (U.S. Govt. Printing Office), 509520.

Estes, R., and Hutchison, J. H., 1980. Eocene lower vertebrates from Ellesmere Island, Canadian Arctic Archipelago. Palaeogeogr., Palaeoclimatol., Palaeoecol., 30:325-347.

Frakes, L. A., and Kemp, E. M., 1973. Palaeogene continental positions and evolution of climate. In Tarling, D. H., and Runcom, S. K. (Eds.), Implication of Continental Drift to the Earth Sciences (Vol. 1): New York (Academic Press), 539-559.

Graham, D. W., Bender, M. L., Williams, D. F., and Keigwin, L. D., 1982. Strontium-calcium ratios in Cenozoic planktonic foraminifera. Geochim. Cosmochim. Acta, 46:1281-1292.

Haq, B. U., Hardenbol, J., and Vail, P. R., 1987. Chronology of fluctuating sea levels since the Triassic. Science, 235:1156-1167.

Hayes, D. E., Frakes, L. A., et al., 1975. Init. Repts. DSDP, 28 : Washington (U.S. Govt. Printing Office).

Hess, J., Stott, L. D., Bender, M. L., Kennett, J. P., and Schilling, J.-G., 1990. The Oligocene marine microfossil record: age assessments using strontium isotopes. Paleoceanography, 4:655-679.

Keigwin, L. D., and Corliss, B. H., 1986. Stable isotopes in late middle Eocene to Oligocene forams. Geol. Soc. Am. Bull., 97:335345.

Keigwin, L. D., and Keller, G., 1984. Middle Oligocene climate change from equatorial Pacific DSDP Site 77. Geology, 12:16-19.

Keller, G., 1983a. Paleoclimatic analyses of middle Eocene through Oligocene planktic foraminiferal faunas. Palaeogeogr., Palaeoclimatol., Palaeoecol., 43:73-94.

1983b. Biochronology and paleoclimatic implications of middle Eocene to Oligocene planktonic foraminiferal faunas. Mar. Micropaleontol., 7:463-468.
Kennett, J. P., 1977. Cenozoic evolution of Antarctic glaciation, the circum-Antarctic Ocean, and their impact on global paleoceanography. J. Geophys. Res., 82:3843-3860.

Kennett, J. P., and Barker, P. F., 1990. Latest Cretaceous to Cenozoic climate and oceanographic developments in the Weddell Sea, Antarctica: an ocean-drilling perspective. In Barker, P. F., Kennett, J. P., et al., Proc. ODP, Sci. Results, 113: College Station, TX (Ocean Drilling Program), 937-960.

Kennett, J. P., and Shackleton, N. J., 1976. Oxygen isotopic evidence for the development of the psychrosphere $38 \mathrm{Myr}$ ago. Nature, 260:513-515.

Kennett, J. P., and Stott, L. D., 1990. Proteus and proto-oceanus: ancestral paleogene oceans as revealed from Antarctic stable isotopic results: ODP Leg 113. In Barker, P. F., Kennett, J. P., et al., Proc. ODP, Sci. Results, 113: College Station, TX (Ocean Drilling Program), 865-880.

Koepnick, R. B., Denison, R. E., and Dahl, D. A., 1988. The Cenozoic Seawater ${ }^{87} \mathrm{Sr} /{ }^{86} \mathrm{Sr}$ Curve: data review and implications for correlation of marine strata. Paleoceanography, 3:743-756.

Lorens, R. B., 1981. Sr, Cd, Mn and Co distribution coefficients in calcite as a function of calcite precipitation rate. Geochim. Cosmochim. Acta., 45:553-561.

Margolis, S. V., and Kennett, J. P., 1971. Cenozoic paleoglacial history of Antarctica recorded in subantarctic deep-sea cores. Am. J. Sci., 271:1-36.

Marty, R., Dunbar, R., Martin, J. B., and Baker, P., 1988. Late Eocene diatomite from the Peruvian coastal desert, coastal upwelling in the eastern Pacific, and Pacific circulation before the terminal Eocene event. Geology, 16:818-822.

Matter, A., Douglas, R. G., and Perch-Nielsen, K., 1975. Fossil preservation, geochemistry and diagenesis of pelagic carbonates from the Shatsky Rise, northwest Pacific. In Larson, R. L., Moberly, R., et al., Init. Repts. DSDP, 32: Washington (U.S. Govt. Printing Office), 891-921.

Matthews, R. K., and Poore, R. Z., 1980. Tertiary $\delta^{18} \mathrm{O}$ record and glacio-eustatic sea-level fluctuations. Geology, 8:501-504.

McGowran, B., and Beecroft, A., 1986. Neritic, southern extratropical foraminifera and the terminal Eocene event. Palaeogeogr., Palaeoclimatol., Palaeoecol., 55:23-34.

Miller, K. G., Aubry, M.-P., Khan, K. J., Melillo, A. J., Kent, D. V., and Berggren, W. A., 1985. Oligocene-Miocene biostratigraphy, magnetostratigraphy and isotopic stratigraphy of the western North Atlantic. Geology, 13:257-261.

Miller, K. G., Fairbanks, R. G., and Mountain, G. S., 1987. Tertiary oxygen isotope synthesis, sea-level history, and continental margin erosion. Paleoceanography, 2:1-19.

Miller, K. G., Feigenson, M. D., Kent, D. V., and Olsson, R. K., 1988. Upper Eocene to Oligocene isotope $\left({ }^{87} \mathrm{Sr}{ }^{86} \mathrm{Sr}, \delta^{18} \mathrm{O}, \delta^{13} \mathrm{C}\right)$ standard section, Deep Sea Drilling Project Site 522. Paleoceanography, 3:223-233.

Miller, K. G., Janecek, T. R., Katz, M. E., and Keil, D. J., 1987. Abyssal circulation and benthic foraminiferal changes near the Paleocene/Eocene boundary. Paleoceanography, 2:741-761.

Miller, K. G., and Thomas, E., 1985. Late Eocene to Oligocene benthic foraminiferal isotopic record, Site 574, equatorial Pacific. In Mayer, L., Theyer, F., et al., Init. Repts. DSDP, 85: Washington (U.S. Govt. Printing Office), 771-777.

Miller, K. G., Wright, J. D., and Brower, A. N., 1989. Oligocene to Miocene stable isotope stratigraphy and planktonic foraminifer biostratigraphy of the Sierra Leone Rise (DSDP Site 366 and ODP Site 667). In Ruddiman, W., Sarnthein, M., et al., Proc. ODP, Sci. Results, 108: College Station, TX (Ocean Drilling Program), 279-294.

Müller, G., and Gastner, M., 1971. The "Karbonat-bombe," a simple device for determination of the carbonate content in sediments, soils and other materials. Neues Jahrb. Mineral. Monatsch., 10:466-469.

Murphy, M. G., and Kennett, J. P., 1986. Development of latitudinal thermal gradients during the Oligocene: oxygen-isotope evidence from the southwest Pacific. In Kennett, J. P., von der Borch, C. C., et al., Init. Repts. DSDP, 90: Washington (U.S. Govt. Printing Office), $1347-1360$.

Mutter, J. C., and Cande, S. C., 1983. The early opening between Broken Ridge and Kerguelen Plateau. Earth Planet. Sci. Lett., 65:369-376. 
North, G. R., 1984. The small ice cap instability in diffusive climate models. J. Atmos. Sci., 41:3390-3395.

Oberhänsli, H., 1986. Latest Cretaceous-early Neogene oxygen and carbon isotopic record at DSDP sites in the Indian Ocean. Mar. Micropaleontol., 10:91-115.

Oberhänsli, H., McKenzie, J. A., Toumarkine, M., and Weissert, H., 1984. A paleoclimatic and paleoceanographic record of the Paleogene in the central South Atlantic (Leg 73, Sites 522, 523, and 524). In Hsü, K. J., LaBrecque, J. L., et al., Init. Repts. DSDP, 73; Washington (U.S. Govt. Printing Office), 737-747.

Poore, R. Z., and Matthews, R. K., 1984. Oxygen isotope ranking of late Eocene and Oligocene planktonic foraminifers: implications for Oligocene sea-surface temperatures and global ice-volume. Mar. Micropaleontol., 9:111-134.

Prentice, J. L., and Matthews, R. K, 1988. Cenozoic ice-volume history: development of a composite oxygen isotope record. Geology, 17:963-966.

Savin, S. M., 1977. The history of the earth's surface temperature during the past one hundred million years. Annu. Rev. Earth. Planet Sci., 5:319-344.

Savin, S. M., Abel, L., Barrera, E., Hodell, D., Kennett, J. P., Murphy, M., Keller, G., Killingley, J., and Vincent, E., 1985. The evolution of Miocene surface and near-surface marine temperatures: oxygen isotopic evidence. In Kennett, J. P. (Ed.), The Miocene Ocean: Paleoceanography and Biogeography. Mem. Geol. Soc. Am., 163:49-82.

Savin, S. M., Douglas, R. G., and Stehli, F. G., 1975. Tertiary marine paleotemperatures. Geol. Soc. Am. Bull., 86:1499-1510.

Schlich, R., Wise, S. W., Jr., et al., 1989. Proc. ODP, Init. Repts., 120: College Station, TX (Ocean Drilling Program).

Schnitker, D., 1980. Quaternary deep sea benthic foraminifers and bottom water masses. Annu. Rev. Earth Planet. Sci., 8:343-370.

Shackleton N. J., and Boersma, A., 1981. The climate of the Eocene ocean. J. Geol. Soc. London, 138:153-157.

Shackleton, N. J., Corfield, R. M., and Hall, M. A., 1985. Stable isotope data and the ontogeny of Paleocene planktonic foraminifera. J. Foraminiferal Res., 15:321-336.

Shackleton, N. J., Hall, M. A., and Boersma, A., 1984. Oxygen and carbon isotope data from Leg 74 foraminifers. In Moore, T. C., Jr.
Rabinowitz, P. D., et al., Init. Repts. DSDP, 74: Washington (U.S. Govt. Printing Office), 599-612.

Shackleton, N. J., and Kennett, J. P., 1975. Paleotemperature history of the Cenozoic and the initiation of Antarctic glaciation: oxygen and carbon isotope analyses in DSDP Sites 277, 279 and 281. In Kennett, J. P., Houtz, R. E., et al., Init. Repts. DSDP, 29: Washington (U.S. Govt. Printing Office), 743-755.

Snyder, S. W., Müller, C., and Miller, K. G., 1984. Eocene-Oligocene boundary: biostratigraphic recognition and gradual paleoceanographic change at DSDP Site 549. Geology, 12:112-115.

Stott, L. D., Kennett, J. P., Shackleton, N. J., Corfield, R. M., 1990. The evolution of Antarctic surface waters during the Paleogene: inferences from stable isotopic composition of planktonic foraminifers, ODP Leg 113. In Barker, P. F., Kennett, J. P., et al., Proc. ODP, Sci. Results, 113: College Station, TX (Ocean Drilling Program), 849-863.

Thomas, E., 1990. Late Cretaceous through Neogene deep-sea benthic foraminifers (Maud Rise, Weddell Sea, Antarctica). In Barker, P. F., Kennett, J. P., et al., Proc. ODP, Sci. Results, 113: College Station, TX (Ocean Drilling Program), 571-594.

Vail, P. R., Mitchum, R. M., and Thompson, S., III, 1977. Seismic stratigraphy and global changes of sea level. Part 4: global cycles of relative changes of sea level. In Payton, C. E. (Ed), Seismic Stratigraphy-Applications to Hydrocarbon Exploration, AAPG Mem., 26:83-97.

Veizer, J., 1978. Simulation of limestone diagenesis-a model based on strontium depletion: discussion. Can J. Earth. Sci., 15:16831686.

Wolfe, J. A., 1980. Tertiary climates and floristic relationships at high latitudes in the northern hemisphere. Palaeogeogr., Palaeoclimatol., Palaeoecol., 30:313-323.

Date of initial receipt: 21 February 1990

Date of acceptance: 20 September 1990

Ms 120B-183 